\title{
Rearing Zebrafish on Black Soldier Fly (Hermetia illucens): Biometric, Histological, Spectroscopic, Biochemical, and Molecular Implications
}

\author{
Arturo Vargas,, Basilio Randazzo,, Paola Riolo,2 Cristina Truzzi, Giorgia Gioacchini,, Elisabetta Giorgini," \\ Nino Loreto, Sara Ruschioni,, Matteo Zarantoniello,' Matteo Antonucci, Sara Polverini, \\ Gloriana Cardinaletti, Simona Sabbatini, Francesca Tulli, and Ike Olivotto ${ }^{1}$
}

\begin{abstract}
A desirable goal of the aquaculture sector is to replace most of fish meal and fish oil with more sustainable, costeffective, and environmental friendly ingredients ensuring fish health and welfare standards. Due to minimal environmental impact, compared with most conventional feed commodities, insects deserve a growing attention as candidate ingredients for aquafeeds. The present study investigated, for the first time, the possible application of a 100\% insect diet in zebrafish larval rearing. Through a multidisciplinary approach, the major biological responses of fish to the new diets were assessed. Results of biometry, fatty acid composition, expression of genes involved in fish growth, stress response, lipid metabolism, chitinolytic activity, gut inflammation, and liver macromolecular composition suggested a possible application of insect larvae for zebrafish larval rearing. However, further studies are necessary to better understand the use of this insect species in the rearing of fish.
\end{abstract}

Keywords: black soldier fly, gene expression, fatty acid profile, insect diet, aquaculture

\section{Introduction}

TN CONTRAST TO world capture fisheries production, which has essentially stagnated since the mid $1980 \mathrm{~s}$, aquaculture has maintained an important annual growth rate worldwide since $2005 \cdot{ }^{1-3}$ Fish meal and fish oil still represent a crucial part of current industrially produced diets, with $\sim 20$ million tons of total marine catches going into the production of aquafeed. $^{2-4}$ Because overexploitation of pelagic fisheries has negative ecological and social consequences, developing a strategy to replace fish meal and fish oil in feeds should become both a private and public-sector priority to reduce pressure and reliance on marine resources while increasing producer profitability. ${ }^{5,6}$ Thus, world-renowned nutritionists and feed technologists are exploring practical ways for the aquaculture industry to expand and remain competitive, and discuss ways to develop less expensive alternative sources of lipid and protein.

Research to develop substitutes for these feed ingredients is now focused on commodities such as oilseeds (especially soybeans), meat byproducts (such as blood meal and bone meal), and microbial proteins. ${ }^{6}$ A move toward partial substitution of plant and terrestrial animal proteins for fish proteins in feed is widely accepted within the aquaculture industry, but the urgency of such efforts remains controversial. Nevertheless, complete replacement of fish meal and fish oil in aquaculture feeds faces severe barriers. ${ }^{4}$ Especially for carnivorous fish, vegetable proteins have inappropriate amino acid balance, poor protein digestibility, and antinutritional substances. ${ }^{7}$ Therefore, inclusion of microalgae ${ }^{8,9}$ and/or meat byproducts can help to overcome this problem.

Recently, a great interest has arisen for the use of insects as a source for feed production, ${ }^{10}$ especially because of their animal origin, their ability of growing on a great variety of organic compounds, including organic wastes, and to the fact that their consumption in animal farming is not in direct competition with human nutrition. ${ }^{11-14}$ Culturing systems are usually simple and require low-cost materials; however, insects' nutritional profiles largely depend on the growing substrate, but also on the selected species and stages of

\footnotetext{
${ }^{1}$ Dipartimento di Scienze della Vita e dell'Ambiente, ${ }^{2}$ Dipartimento di Scienze Agrarie Alimentari ed Ambientali, Università Politecnica delle Marche, Ancona, Italy.

${ }^{3}$ Dipartimento di Scienze Agroalimentari, Ambientali e Animali, Università di Udine, Udine, Italy.

${ }^{4}$ Dipartimento di Scienze e Ingegneria della Materia, dell'Ambiente ed Urbanistica, Ancona, Italy.
} 
development, ${ }^{15-17}$ underlying the need for further studies to better understand their possible application in the aquaculture field. For example, the study by Barroso and collaborators ${ }^{16}$ evidenced a poor $\mathrm{n}-3$ and $\mathrm{n}-6$ fatty acid content in 16 different insect species belonging to Coleoptera, Diptera, and Orthoptera order. However, ST-Hilaire and collaborators ${ }^{18}$ showed that the use of fish offal as growing substrate, along with cow manure, enhanced the lipid profile of the black soldier fly (BSF), Hermetia illucens (L.) (Diptera, Stratiomyidae), in terms of omega-3 fatty acids.

Insects may be carriers of biological and chemical hazards, such as prions, viruses, parasites, organic contaminants (e.g., dioxin compounds and pesticides), and heavy metals. ${ }^{19,20}$ Proper management and prevention during insect production is therefore a must; in fact, a recent study suggested that preventive techniques similar to that of other farmed animals should guarantee food safety of edible insects. ${ }^{20}$

Insects are characterized by having an exoskeleton of chitin. Fish have limited digestive capacity for chitin and it has been demonstrated that diets rich in chitin can have a negative impact on food intake, availability, digestibility, and absorption of nutrients and thus growth performances. ${ }^{21}$

Among the different insect species that have been cultured and studied for their possible application in the aquaculture field, BSF represents one of the most interesting ones. ${ }^{18,22}$ Rearing BSF has been proposed since the $1990 \mathrm{~s}$ as an efficient way for converting organic wastes into a protein-rich and fat-rich biomass suitable for various purposes, including animal feeding. ${ }^{23}$ It is an extremely resistant species and the larvae process organic wastes very quickly. ${ }^{23}$ The adult does not feed and therefore does not require particular care and is not a potential carrier of diseases. ${ }^{23}$ However, its culture requires a warm environment, which may be difficult or energy consuming to sustain in temperate climates. Nowadays, feeds based on BSF larvae open additional marketing opportunities in the aquaculture sector. ${ }^{10}$

Future growth and profitability within the aquaculture sector is dependent upon continued improvements in diet efficiency and formulation; specifically a reduction or even a complete substitution of unsustainable marine protein and lipid feedstuff is desirable and insects may thus represent an ideal alternative. In line with this, the aim of the present study was to evaluate the suitability of a $100 \%$ insect diet, based on BSF grown on two different substrates, on zebrafish larval development through a multidisciplinary approach, including morphological, biochemical, spectroscopic, and molecular analysis. Zebrafish represents an extraordinary experimental model for biomedical, developmental biology, genetics, toxicology, and aquaculture studies, due to its high reproductive rate and to the abundant information that has recently become available from genomic sequencing. ${ }^{24,25}$ Particularly, zebrafish are used to generalize how several biological processes occur in related organisms, and contribute toward improving our understanding of the mechanisms involved in nutrition and growth. ${ }^{26-29}$

\section{Materials and Methods}

\section{Ethics}

All procedures involving animals were conducted in line with Italian legislation on experimental animals and were approved by the Ethics Committee of the Università Poli- tecnica delle Marche. Optimal rearing conditions (see further section for details) were applied throughout the study, and all efforts were made to minimize animal suffering by using an anesthetic (MS222; Sigma-Aldrich).

\section{Insect sources}

Significant amounts of BSF were cultured at different conditions as follows:

Group A: BSF were reared on byproducts obtained from roasting coffee process [provided by Saccaria Caffè S.R.L., Marina di Montemarciano (AN), Italy]. BSF laboratory colony was originated from commercially available prepupae [Smart Bugs s.s. company, Ponzano Veneto (TV), Italy]. The colony was maintained under controlled conditions $\left(30^{\circ} \mathrm{C} \pm\right.$ $2^{\circ} \mathrm{C}, 70 \% \pm 10 \% \mathrm{RH}$ and 14:10L:D). Prepupae were placed in different plastic boxes $(57 \times 39 \times 28 \mathrm{~cm} / 45 \mathrm{~L})$ until pupa stage was reached. Emerged adults were placed in $2 \mathrm{~m}^{3}$ cages and supplied with water and sugar ad libitum and, $\sim 2$ days after emergence, coffee byproduct was provided as egg-laying substrate. The coffee substrate was placed in small plastic boxes $(16.5 \times 22 \mathrm{~cm})$ providing the females suitable sites for egg laying (presence of crevices). The plastic boxes were changed every 2 days. Three thousand 4-day-old larvae (which were individually counted with the aid of entomological tweezers and a brush to avoid damaging the larvae) were transferred in each of the plastic boxes $(57 \times 39 \times 28 \mathrm{~cm} / 45 \mathrm{~L})$ and fed with coffee byproducts. The feeding substrate was supplied ad libitum to the developing larvae. ${ }^{30}$ Larval rearing boxes were equipped with a ramp $\left(35^{\circ}\right)$ and a collection device attached to the end of the ramp. When the larvae reached the prepupal stage, they left the feeding substrate, climbed up the ramp to end in the collecting vessel, as they migrated away from the feeding substrate to pupate. The life cycle, from egg to prepupae, occurred approximately in 4 months.

Group B: BSF prepupae were purchased from a commercial company [Smart Bugs s.s. company, Ponzano Veneto (TV), Italy]. Insects were fed on a feeding substrate composed of corn meal and fruit and vegetable mixture (50:50).

All prepupae (group A and group B), once collected, were frozen $\left(\right.$ at $\left.-80^{\circ} \mathrm{C}\right)$, freeze dried, minced, and sieved $(300 \mu \mathrm{m})$.

\section{Fish feeding trial}

Zebrafish AB embryos were spawned and maintained $48 \mathrm{~h}$ in a Tecniplast system (Varese, Italy), subjected to the following conditions: $\mathrm{pH} 7 ; \mathrm{NO}_{2}$ and $\mathrm{NH}_{3}<0.01 \mathrm{mg} \mathrm{L}^{-1} ; \mathrm{NO}_{3}$ $<10 \mathrm{mg} \mathrm{L}^{-1}$; and photoperiod12 L:12 D. After this first period, embryos were gently collected, counted under a stereomicroscope (Leica Wild M3B; Leica Microsystems), and divided in nine experimental tanks (200 larvae each in 20-L tanks) that were randomly assigned to the three dietary treatments (each in triplicate). The water in the 20-L larval tank (same chemical-physical characteristics of the parent's tank) was gently replaced ten times a day by a dripping system. The sides of the tank were covered with black panels to reduce light reflection. ${ }^{31,32}$

Control group: larvae fed a commercial feed (Blue Line, Italy; for composition www.blue-co.it/alimenti-in-pellets. html); group A: larvae fed the BSF grown on byproducts obtained from roasting coffee process (Proximate composition\%: Crude protein: $34.7 \pm 2.2$; Ether Extract: $18.8 \pm 0.5$; 
Ash: $10.0 \pm 0.4$; and Nitrogen-Free extract: $36.4 \pm 4.2$ ); group B: larvae fed on the BSF grown on corn meal, and fruit and vegetable mixture (50:50) (Proximate composition\%: Crude protein: $36.7 \pm 4.2$; Ether Extract: $16.8 \pm 0.8$; Ash 11.8 \pm 0.3 ; and Nitrogen-Free extract: $34.6 \pm 3.2$ ). All groups were fed on the rotifer Brachionus plicatilis ( $5 \mathrm{ind} / \mathrm{mL}$ ) from 5 to 10 days postspawning (dps; one feeding in the morning) according to Lawrence and collaborators. ${ }^{33}$ Zebrafish larvae were maintained in $20-\mathrm{L}$ tanks and fed the experimental diets at $2 \%$ body weight, twice a day. ${ }^{24,25}$ Larvae were sampled at 7,14 , and $21 \mathrm{dps}$, euthanized with an excess of anesthetic (MS222 $1 \mathrm{~g} / \mathrm{L}$; Sigma), and properly stored for further analysis.

\section{Biometry and survival}

At each sampling time, 10 zebrafish larvae, in triplicate, were randomly collected from the different tanks. The standard length was estimated through the use of a caliper (Measy, New Zealand) (precision $0.1 \mathrm{~mm}$ ) and the dry weight (calculated in pools of ten larvae). Larvae were rinsed with fresh distilled water, placed in individual, preweighed capsules, dried at $60^{\circ} \mathrm{C}$ for at least $24 \mathrm{~h}$, and finally weighed (METTLER TOLEDO microgram balance UMX2 Automated$\mathrm{S}$ ultra-microbalance, (Columbus, $\mathrm{OH}$ ) and $\mathrm{UM} 3$ precision single-pan balance, Switzerland). The specific growth rate (SGR) for each experimental group/tank was calculated as follows:

$$
\mathrm{SGR} \%=((\ln W f-\ln W i) / t) \times 100,
$$

where $W f$ is the final dry weight ( $21 \mathrm{dps}), W i$, the initial dry weight ( $7 \mathrm{dps}$ ), and $t$, the number of days (14). Finally, survival was determined by counting the dead and alive larvae (in triplicate) at the end of the experiment ( $21 \mathrm{dps}$ ).

\section{Lipid extraction and fatty acid analysis}

BSF feeding substrates (group A and group B), experimental diets (control group, group A, and group B), and larval fish samples collected at $21 \mathrm{dps}$ (control group, group A, and group B), each in triplicate, were minced, homogenized (MZ 4110; DCG Electronic), and freeze dried (Edwards EF4; Crawley, Sussex, England). Lipid extraction was carried out on lyophilized powders following a Microwave-Assisted Extraction (MAE). ${ }^{34,35}$ Fatty acid methyl esters (FAMEs) were prepared according to Truzzi and collaborators (2017), using the methyl ester of nonadecanoic acid (19:0, Dr. Ehrenstorfer GmbH; Germany) as internal standard. FAMEs were determined on an Agilent-6890 GC System coupled to an Agilent-5973 N quadrupole mass selective detector. A CPS ANALITICA CC-wax-MS $(30 \mathrm{~m} \times 0.25 \mathrm{~mm}$ ID, $0.25 \mu \mathrm{m}$ film thickness $)$ capillary column was used to separate FAMEs. Instrumental conditions were set up as in Truzzi and collaborators, ${ }^{34}$ and no overlapping between peaks were noted. The mass fraction of fatty acids as $\mathrm{mg} \mathrm{g}^{-1}$ tissue dry weight (dw) was measured against internal standard. For each sample, at least three runs were performed on the GC-MS.

The precision of the proposed method was evaluated for the studied matrices as in Truzzi and collaborators, ${ }^{36}$ and it was similar to that found for fish muscle. ${ }^{34}$ For insect substrates, experimental diets and zebrafish larvae, the intraday and interday precision were, for major FAs, $<4 \%$ and $<9 \%$, respectively, indicating a good repeatability of the analyses (data not shown). For FAs with a percentage $<1 \%$ versus total FAs, intraday and interday precision ranged from $6 \%$ to $20 \%$, and from $7 \%$ to $25 \%$, respectively. The estimated limits of detection and limits of quantification, calculated as in Truzzi and collaborators, ${ }^{37}$ ranged for each FAME from $\sim 4$ to $\sim 22 \mu \mathrm{g}$ $\mathrm{mL}^{-1}$, and from $\sim 13$ to $\sim 66 \mu \mathrm{g} \mathrm{mL}^{-1}$, respectively. ${ }^{34}$

\section{Histology}

Ten zebrafish larvae, in triplicate, randomly collected at each sampling time from the different tanks, were fixed by immersion in $4 \%$ paraformaldehyde and stored at $4{ }^{\circ} \mathrm{C}$ for $24 \mathrm{~h}$. Larvae were washed three times with PBS $0.1 \mathrm{M}(\mathrm{pH}$ 7.4) buffer for $10 \mathrm{~min}$ and preserved in ethanol (70\%). Larvae were then dehydrated by subsequent washing in ethanol $(80 \%, 95 \%$, and $100 \%)$, washed with clearing agent "HistoClear" (Bio-Clear, Bio Optica) and embedded in liquid paraffin (Bio-Optica, Milano, Italy) at $55-58^{\circ} \mathrm{C}$. Solidified paraffin blocks were cut with a microtome (Leica RM2125 RTS) to obtain $5 \mu \mathrm{m}$ sections then stained with Hematoxylin (Mayer) and Eosin Y (Sigma-Aldrich). Sections were observed using a Leica MD750 optical microscope connected with a camera Leica ICC50 HD.

\section{Fourier transform infrared microspectroscopy measurements and data analysis}

Intestines from five different specimens of 21 dps zebrafish larvae fed BSF diet (group A and group B) and a commercial feed (control group), were quickly dissected and immediately frozen at $-80^{\circ} \mathrm{C}$. Then, each intestine sample was cut by using a cryomicrotome (Microm HM $505 \mathrm{~N}$ ) to obtain six thin adjacent sections $(\sim 10 \mu \mathrm{m}$ thick): three sections were deposited onto $\mathrm{CaF}_{2}$ optical windows $(1 \mathrm{~mm}$ thick, $13 \mathrm{~mm}$ diameter) for Fourier transform infrared microspectroscopy (FTIRM) analysis, whereas the other three sections were placed onto conventional glass slides for morphological examination (Hematoxylin and Eosin staining) ${ }^{38}$

FTIR microspectroscopy studies the interaction between the infrared radiation and the chemical bonds and couples the diagnostic potential of IR spectroscopy with the visual inspection performed by Vis microscopy. It lets analyze nonhomogeneous tissue sections by acquiring on the same sample and at the same time the microphotograph and the IR map, which represents the distribution of the total absorption of the infrared radiation on the mapped area. For each pixel of the map, it is possible to retrieve the corresponding vibrational signature, and hence the macromolecular building and composition of the sample..$^{39,40}$

FTIRM analysis was performed at room temperature on 45 sections (15 for each experimental group) within $24 \mathrm{~h}$ from cutting. Intestine sections prepared by using this protocol have been already tested in previous experiments in our laboratory, evidencing a good stability in time and providing homogeneous and reliable vibrational data sets. A PerkinElmer Spectrum GXI Spectrometer, equipped with a PerkinElmer AutoIMAGE microscope and a photoconductive $\mathrm{HgCdTe}$ MCT array detector, operating at liquid nitrogen temperature, which covers the entire IR spectral range from 4000 to $700 \mathrm{~cm}^{-1}$, was used.

By means of a microscope television camera, specific areas were selected on each section, according with the histological 
suggestions. On these areas, the IR maps $\left(\sim 300 \times 500 \mu \mathrm{m}^{2}\right)$ were acquired in transmission mode in the MIR range from 4000 to $800 \mathrm{~cm}^{-1}$ (spectral resolution $4 \mathrm{~cm}^{-1}$, spatial resolution $40 \times 40 \mu \mathrm{m}^{2}$ and 128 scans). Background spectra were acquired on clean portions of the $\mathrm{CaF}_{2}$ optical windows and compared against samples spectra. IR maps are false color images representing the total intensity of the infrared absorption; each pixel, whose dimensions correspond to the spatial resolution, is represented by a single IR spectrum. IR maps were automatically corrected for avoiding the contributions of carbon dioxide and water vapor and vector normalized on the full frequency range (to avoid artifacts due to local thickness variations). Spectrum Image 5.1.0 (PerkinElmer) and Spectrum 10.4 (PerkinElmer) software were used for data handling.

From each map, 50 spectra were selected and vector normalized. Then, the area integrals of the following spectral regions were calculated: $3000-2815 \mathrm{~cm}^{-1}$ (corresponding to the symmetric and asymmetric stretching modes of $\mathrm{CH}_{2}$ and $\mathrm{CH}_{3}$ groups, representative of lipids, named LIP); 1771$1715 \mathrm{~cm}^{-1}$ (corresponding to the stretching mode of $\mathrm{C}=\mathrm{O}$ group of fatty acids, named FA); $1715-1481 \mathrm{~cm}^{-1}$ (corresponding to Amide I and Amide II bands of proteins, named PRT); $1481-1425 \mathrm{~cm}^{-1}$ (corresponding to $\mathrm{CH}_{2}$ groups of alkyl chains, named $\mathrm{CH} 2$ ), and $1143-985 \mathrm{~cm}^{-1}$ (corresponding to glycosylated proteins, and mainly mucin, named MUCIN). The area integrals of the $2998-2800 \mathrm{~cm}^{-1}$ and $1775-950 \mathrm{~cm}^{-1}$ were added, and their sum was considered representative of the total tissue biomass (TBM). The aforementioned integrals were used to calculate the following band area ratios: LIP/TBM, FA/TBM, CH2/TBM, PRT/ TBM, and MUCIN/TBM.

\section{RNA extraction and CDNA synthesis}

Total RNA extraction from 20 zebrafish larvae (in triplicate), randomly collected from the different tanks at each sampling point, was optimized using RNAzol ${ }^{\circledR}$ RT reagent (Sigma-Aldrich ${ }^{\circledR}, \mathrm{R} 4533$ ) following the manufacturer's instructions. RNA was then eluted in $20 \mu \mathrm{L}$ of RNase-free water. Final RNA concentration was determined by the NanoPhotometer ${ }^{\circledR}$ P-Class (Implen, Germany). RNA integrity was verified by GelRed ${ }^{\mathrm{TM}}$ staining of $28 \mathrm{~S}$ and $18 \mathrm{~S}$ ribosomal RNA bands on $1 \%$ agarose gel. RNA was stored at $-80^{\circ} \mathrm{C}$ until use. Finally, $3 \mu \mathrm{g}$ of RNA was used for cDNA synthesis, employing the High-Capacity cDNA Reverse Transcription Kit (Bio-Rad) following the manufacturer's instructions.

\section{Real-time PCR}

PCRs were performed with SYBER Green in an iQ5 iCycler thermal cycler (both from Bio-Rad) in triplicate. Reactions were set on a 96-well plate by mixing for each sample $1 \mu \mathrm{L}$ cDNA diluted $1: 20,5 \mu \mathrm{L}$ of $2 \times$ concentrated $\mathrm{iQ}^{\mathrm{TM}}$ SYBER Green as the fluorescent intercalating agent, $0.3 \mu \mathrm{M}$ forward primer, and $0.3 \mu \mathrm{M}$ reverse primer. The thermal profile for all reactions was $3 \mathrm{~min}$ at $95^{\circ} \mathrm{C}$, followed by 45 cycles of $20 \mathrm{~s}$ at $95^{\circ} \mathrm{C}, 20 \mathrm{~s}$ at $60^{\circ} \mathrm{C}$, and $20 \mathrm{~s}$ at $72^{\circ} \mathrm{C}$. Fluorescence was monitored at the end of each cycle. Dissociation curve analysis showed a single pick in all cases.

Relative quantification of the expression of genes involved in fish growth (igfl, igf $2 a$, and $m s t n b)$, fatty acid desaturation (fad2) and elongation (elovl5 and elovl2), stress response (nr3cland hsp70.1), and enzymatic hydrolysis of chitin (chia.1, chia.2, chia.3, chia.4, chia.5, and chia.6) was performed using rplpO and rpll3 as housekeeping genes to standardize the results (Table 1).

Amplification products were sequenced and homology was verified. No amplification product was detected in negative controls and no primer-dimer formation was found in control templates. Data were analyzed using the iQ5 optical system software version 2.0, including Genex Macro iQ5 Conversion and Genex Macro iQ5 files (all from Bio-Rad). Modification of gene expression is reported with respect to controls. Primer sequences were designed using Primer3 (210 v. 0.4.0) starting from zebrafish sequences available in ZFIN. Primers were used at a final concentration of $10 \mathrm{pmol} / \mu \mathrm{L}$.

\section{Statistics}

Survival, SGR, and FTIRM data were analyzed by oneway ANOVA, with diet as the explanatory variable. Lipid content and fatty acid data were analyzed by both $t$-test and one-way ANOVA. Standard length, dry weight, and real-time

Table 1. Primer Sequences and ZFID Used in the Present Study

\begin{tabular}{llll}
\hline Gene & \multicolumn{1}{c}{ Forward primer $\left(5^{\prime}-3^{\prime}\right)$} & \multicolumn{1}{c}{ Reverse primer $\left(5^{\prime}-3^{\prime}\right)$} & \multicolumn{1}{c}{ ZFIN ID } \\
\hline igfl & GGCAAATCTCCACGATCTCTAC & CGGTTTCTCTTGTCTCTCTCAG & ZDB-GENE-010607-2 \\
igf2a & GAGTCCCATCCATTCTGTTG & GTGGATTGGGGTTTGATGTG & ZDB-GENE-991111-3 \\
mstnb & GGACTGGACTGCGATGAG & GATGGGTGTGGGGATACTTC & ZDB-GENE-990415-165 \\
nr3c1 & AGACCTTGGTCCCCTTCACT & CGCCTTTAATCATGGGAGAA & ZDB-GENE-050522-503 \\
hsp70.1 & TGTTCAGTTCTCTGCCGTTG & AAAGCACTGAGGGACGCTAA & ZDB-GENE-990415-91 \\
fads2 & CATCACGCTAAACCCAACA & GGGAGGACCAATGAAGAAGA & ZDB-GENE-011212-1 \\
elovl5 & TGGATGGGACCGAAATACAT & GTCTCCTCCACTGTGGGTGT & ZDB-GENE-040407-2 \\
elovl2 & CACTGGACGAAGTTGGTGAA & GTTGAGGACACACCACCAGA & ZDB-GENE-060421-5612 \\
chia.1 & ACTGGGCGGAGCCTCAGTGT & GGGCTTGGGTGGGAAACCCAG & ZDB-GENE-040426-1994 \\
chia.2 & GGTGCTCTGCCACCTTGCCTT & GGCATGGTTGATCATGGCGAAAGC & ZDB-GENE-040426-2014 \\
chia.3 & TCGACCCTTACCTTTGCACACACCT & ACACCATGATGGAGAACTGTGCCGA ZDB-GENE-040426-2891 \\
chia.4 & TGGACACCTCCACACGCTGC & ATGCCCACTAATCCGCCCGC & ZDB-GENE-030131-9279 \\
chia.5 & CCACGGCTCACAGGACAACATCA & GTCCGCAGACGACAGGCGAA & ZDB-GENE-071004-113 \\
chia.6 & TCCACGGCTCATGGGAGAGTGTC & AGCGCCTGGTCTCGCCAGT & ZDB-GENE-030131-1140 \\
rplp0 & CTGAACATCTCGCCCTTCTC & TAGCCGATCTGCAGACACAC & ZDB-GENE-000629-1 \\
rpli3 & TCTGGAGGACTGTAAGAGGTATGC & AGACGCACAATCTTGAGAGCAG & ZDB-GENE-031007-1 \\
\hline
\end{tabular}


PCR data were analyzed by two-way ANOVA, with both diet and dps as the explanatory variables. Normality and homogeneity of variance was verified through residual plots according to Zuur and collaborators, ${ }^{41}$ revealing no violations of ANOVA and $t$-test assumptions. All ANOVA tests were followed by Tukey's posttest. The statistical software package Prism5 (GraphPad Software) was used; significance was set at $p<0.05$. All results are presented as mean \pm SD.

\section{Results}

\section{Biometry}

At the end of the experiment ( $21 \mathrm{dps}$ ), the survival of the zebrafish larvae did not exhibit significant statistical differences among the dietary treatments $(p>0.05)$, with control group showing the highest value $(44 \% \pm 14 \%)$ with respect to group $\mathrm{A}$ and $\mathrm{B}(38 \% \pm 20 \%$ and $30 \% \pm 5 \%$, respectively) (Table 2).

As regards the standard length, no significant differences $(p>0.05)$ among the experimental groups were detected at all sampling times $(7,14$, and $21 \mathrm{dps}$; Table 2$)$. At the end of the experiment, control group larvae showed the highest standard length $(6.5 \pm 1.2 \mathrm{~mm})$, whereas group A and group B showed lower standard length with respect to control group $(5.7 \pm 0.8 \mathrm{~mm}$ and $5.4 \pm 1.1 \mathrm{~mm}$, respectively; Table 2$)$. As regards dry weight, no significant differences $(p>0.05)$ were observed among the three experimental groups, at all experimental times $(7,14,21 \mathrm{dps}$; Table 2). At $21 \mathrm{dps}$ control group larvae showed the highest dry weight $(4.5 \pm 2.3 \mathrm{mg})$, whereas group A and group B showed a $2.3 \pm 0.8 \mathrm{mg}$ and $1.8 \pm 0.7 \mathrm{mg}$ dry weight, respectively (Table 2 ). However, no statistical differences were observed $(p>0.05)$. Finally, considering SGR, control group larvae showed the highest value (27.1 \pm 3.5$)$, whereas group A and group B showed a 22.4 \pm 2.6 and 19.5 \pm 4.6 SGR, respectively (Table 2); no significant differences were detected $(p>0.05)$.

\section{Lipid content}

Table 3 shows the dry weight (dw) and lipid percentage of BSF feeding substrates, experimental diets, and zebrafish larvae collected at $21 \mathrm{dps}$. Group A substrate showed dry weight significantly lower $(p<0.05)$ than group B substrate $(52.3 \pm 0.9$ and $89.6 \pm 0.4$, respectively). Lipid percentage of substrates showed no significant differences (group A $3.4 \% \pm 0.1 \%$ and group B $3.0 \% \pm 0.7 \% ; p>0.05)$. As regards the diets, both control group and group $\mathrm{A}$, showed a signifi- cantly $(p<0.05)$ higher dry weight $(97.6 \pm 0.2 \mathrm{mg}$ and $98.6 \pm$ $0.3 \mathrm{mg}$, respectively) with respect to group B $(92.4 \pm 0.6 \mathrm{mg})$. Lipid percentage of control group and group A diet showed no significant differences $(11 \% \pm 1 \%$ and $13 \% \pm 1 \%$, respectively; $p>0.05$ ), whereas group B diet showed a significantly higher lipid percentage $(25 \% \pm 1 \% ; p<0.05)$ with respect to both control group and group A. Considering zebrafish larvae, group A samples $(23.6 \% \pm 3.2 \%)$ did not show significant differences $(p>0.05)$ in dw percentage with respect to control group $(16.7 \% \pm 2.7 \%)$. Group B larvae $(15.9 \% \pm 3.0 \%)$ showed a dry weight percentage significantly lower $(p<0.05)$ than group A. As regards the lipid percentage of the larvae, no significant differences $(p>0.05)$ among experimental groups were observed (control group 9.5 \pm 1.8 , group A 7.3 \pm 3.2 and group B $12.7 \% \pm 3.8 \%$; Table 3 ).

\section{Fatty acids composition}

Table 4 reports the FA composition (as $\mathrm{mg} \mathrm{g}^{-1} \mathrm{dw}$ ) of the insect substrates, the experimental diets, and zebrafish larvae collected at $21 \mathrm{dps}$.

Both insect substrates were characterized by a high content of palmitic acid (16:0) that, with stearic acid (18:0), represented more than $70 \%$ of saturated fatty acid (SFA). Among monounsaturated fatty acid (MUFA), oleic acid (18:1n9) was the most important; polyunsaturated fatty acid (PUFA) was not present, with the exception of linoleic acid (18:2n6) and $\alpha$-linolenic acid (18:3n3).

As regards the diets, group A and group B showed a very high content of lauric acid (12:0) that represented about 55\% of SFA, and $\sim 40 \%$ of the total FAs, for both groups. On the contrary, SFA of control group diet were characterized by a high content of palmitic acid (16:0), followed by stearic acid (18:0) and myristic acid (14:0). The most important MUFA was oleic acid $(18: 1 n 9)$ for all experimental diets; palmitoleic acid $(16: 1 n 7)$ was also present in significant quantity. Control group diet showed a high content of omega-3 EPA (eicosa-5,8,11 ,14,17-pentaenoic acid, 20:5n3), DHA (docosa-4,7,10,13,16, 19-hexaenoic acid, 22:6n3), and linoleic acid (18:2n6), whereas group A and group B showed a high content of linoleic acid (18:2n6), but did not show PUFA with $\mathrm{C}$ number $>18$, by consequence they were poor in $n 3$ FAs.

The control group of zebrafish larvae showed a high content of palmitic acid (16:0), followed by stearic and myristic acids (14:0); in addition, group A and group B also showed a high content of lauric acid (12:0). The most represented MUFA was the same detected in the diets, with oleic acid

Table 2. Survival, Standard Length (mm), Dry Weight (mg), and Specific Growth Rate (SGR\% Increase in Dry Weight Day ${ }^{-1}$ ) of Zebrafish Larvae Fed Two Different Black Soldier Fly Diets (Group A and Group B) and a Commercial Feed (Control)

\begin{tabular}{|c|c|c|c|c|c|c|c|c|c|}
\hline & \multicolumn{3}{|c|}{ Control } & \multicolumn{3}{|c|}{ Group A } & \multicolumn{3}{|c|}{ Group B } \\
\hline & $7 d p s$ & $14 d p s$ & $21 d p s$ & $7 d p s$ & $14 d p s$ & $21 d p s$ & $7 d p s$ & $14 d p s$ & $21 d p s$ \\
\hline Survi & - & - & $44 \pm 1$ & - & - & $38 \pm 20$ & - & - & $30 \pm 5$ \\
\hline length & $3.2 \pm 0.7$ & $4.5 \pm 0.7$ & & $3.2 \pm 0.3$ & $4.6 \pm 0.5$ & & $3.5 \pm 0.2$ & $4.2 \pm 0.3$ & \\
\hline Dry weight & $0.1 \pm 0.0$ & $1.2 \pm 0.3$ & $4.8 \pm 2.3$ & $0.1 \pm 0.0$ & $0.6 \pm 0.0$ & $2.4 \pm 0.8$ & $0.1 \pm 0.0$ & $1.2 \pm 0.4$ & $1.9 \pm 0.7$ \\
\hline SGR & - & - & $28.4 \pm 1.8$ & - & - & $22.5 \pm 2.6$ & - & - & $19.8 \pm 4.6$ \\
\hline
\end{tabular}

No significant differences among groups were detected $(p>0.05)$.

SGR, specific growth rate. 
Table 3. Dry Weight and Lipid (Dry Matter Basis) Percentage in the Insect Substrates, Experimental Diets, and Zebrafish Larvae Collected at 21 dPS

\begin{tabular}{|c|c|c|c|c|c|c|c|c|}
\hline & \multicolumn{2}{|c|}{ Insect substrate } & \multicolumn{3}{|c|}{ Experimental diet } & \multicolumn{3}{|c|}{ Zebrafish larvae } \\
\hline & Group A & Group B & Control & Group A & Group B & Control & Group A & Group B \\
\hline$\%$ dry weight & $52.3 \pm 0.9$ & $89.6 \pm 0.4^{\mathrm{a}}$ & $97.6 \pm 0.2$ & $98.6 \pm 0.3$ & $92.4 \pm 0.6^{\mathrm{a}}$ & $16.7 \pm 2.7$ & $23.6 \pm 3.2$ & $15.9 \pm 3.0^{\mathrm{a}}$ \\
\hline$\%$ lipid dw & $3.4 \pm 0.1$ & $3 \pm 0.7$ & $11 \pm 1.0$ & $13 \pm 1.0$ & $25 \pm 1.0^{\mathrm{a}}$ & $9.5 \pm 1.8$ & $7.3 \pm 3.2$ & $12.7 \pm 3.8$ \\
\hline
\end{tabular}

${ }^{a}$ Indicates statistically significant difference among experimental groups $(p<0.05) . N=3$.

showing the highest content, followed by palmitoleic acid and vaccenic acid (18:1n7). About PUFA, control group showed high content of EPA, DHA, and linoleic acid (18:2n6); group A and B showed a high content of linoleic acid and they also showed a significant $(p<0.05)$ content of DHA.

Figure 1 reports the composition (as $\mathrm{mg} \mathrm{g}^{-1} \mathrm{dw}$ ) of SFA, MUFA, and PUFA, as well as the omega-3 (n3), omega-6 (n6), and omega-9 (n9) content, and the n6/n3 ratio in the insect substrates (Fig. 1a), the experimental diets (Fig. 1b), and zebrafish larvae collected at $21 \mathrm{dps}$ (Fig. 1c).

Substrates. Group A substrate was characterized by a remarkably higher concentration of SFA $(p<0.05 ; 40.7 \pm$ $\left.13.9 \mathrm{mg} \mathrm{g}^{-1} \mathrm{dw}\right)$ and a lower PUFA content $(p<0.05$; $0.7 \pm 0.1 \mathrm{mg} \mathrm{g}^{-1} \mathrm{dw}$ ) compared with group B substrate (SFA $1.3 \pm 0.3 \mathrm{mg} \mathrm{g}^{-1} \mathrm{dw}$, PUFA $2.8 \pm 0.7 \mathrm{mg} \mathrm{g}^{-1} \mathrm{dw}$, respectively; Fig. 1a). Group A substrate showed significantly $(p<0.05)$ less n6 $\left(0.7 \pm 0.1 \mathrm{mg} \mathrm{g}^{-1} \mathrm{dw}\right)$ with respect to group B substrate $\left(2.3 \pm 0.5 \mathrm{mg} \mathrm{g}^{-1} \mathrm{dw}\right)$ and a higher content of $\mathrm{n} 9(3.7 \pm 1.3 \mathrm{mg}$ $\left.\mathrm{g}^{-1} \mathrm{dw}\right)$ with respect to group B substrate $\left(0.9 \pm 0.2 \mathrm{mg} \mathrm{g}^{-1}\right.$ dw). Unlike group B, group A did not show n3 FAs. As regards the $\mathrm{n} 6 / \mathrm{n} 3$ ratio, no significant differences $(p<0.05)$ between substrates were observed.

Diets. Both group A and group B diets showed a significantly $(p<0.05)$ higher SFA content $(50.1 \pm 2.8$ and 80.2 \pm $6.2 \mathrm{mg} \mathrm{g}^{-1} \mathrm{dw}$, respectively) and a significantly $(p<0.05)$ lower MUFA content $\left(16.0 \pm 0.4 \mathrm{mg} \mathrm{g}^{-1} \mathrm{dw}\right.$ and $21.9 \pm$ $0.8 \mathrm{mg} \mathrm{g}^{-1} \mathrm{dw}$, respectively) with respect to control group (SFA $29.3 \pm 1.4 \mathrm{mg} \mathrm{g}^{-1} \mathrm{dw}$ and MUFA $24.9 \pm 1.2 \mathrm{mg} \mathrm{g}^{-1} \mathrm{dw}$ ). Regarding the PUFA content, both group A and group B diets showed a significantly $(p<0.05)$ lower content $(4.6 \pm 0.1$ and $8.7 \pm 0.3 \mathrm{mg} \mathrm{g}^{-1} \mathrm{dw}$, respectively) with respect to control group (50.8 $\pm 1.5 \mathrm{mg} \mathrm{g}^{-1} \mathrm{dw}$; Fig. $\left.1 \mathrm{~b}\right)$.

Table 4. Fatty Acid Composition (as Mg G ${ }^{-1}$ DW) of Insect Substrates, Experimental Diets, AND ZeBrafish LaRvae COLleCted AT 21 DPS

\begin{tabular}{|c|c|c|c|c|c|c|c|c|}
\hline & \multicolumn{2}{|c|}{ Insect substrates } & \multicolumn{3}{|c|}{ Experimental diets } & \multicolumn{3}{|c|}{ Zebrafish larvae } \\
\hline & Group A & Group B & Control & Group A & Group B & Control & Group A & Group B \\
\hline $10: 0$ & $=0.06$ & 0.001 & $<\mathrm{DL}$ & $1.41 \pm 0.06^{\mathrm{a}}$ & $2.30 \pm 0.2^{\mathrm{b}}$ & $<\mathrm{DL}$ & $0.07 \pm$ & $0.12 \pm 0.02^{\mathrm{a}}$ \\
\hline $12: 0$ & $0.20 \pm 0.07$ & $.026 \pm 0.008$ & $0.16 \pm 0.04^{\mathrm{a}}$ & & $44 \pm 4^{\mathrm{c}}$ & $1.18 \pm 0.40^{\mathrm{a}}$ & $8.3 \pm 1.1^{\mathrm{b}}$ & $21 \pm 2^{\mathrm{c}}$ \\
\hline $13: 0$ & $0.03 \pm 0.01$ & $<\mathrm{DL}$ & $0.051 \pm 0.007^{\mathrm{a}}$ & $0.106 \pm 0.004^{\mathrm{a}}$ & $0.059 \pm 0.003^{\mathrm{a}}$ & $0.041 \pm 0.008^{\mathrm{a}}$ & $0.06 \pm 0.02^{\mathrm{a}}$ & $0.07 \pm 0.01^{\mathrm{a}}$ \\
\hline $14: 0$ & $3.1 \pm 1.2$ & $.045 \pm 0.007 *$ & $4.9 \pm 0.8^{\mathrm{a}}$ & $8.2 \pm 0.9^{\mathrm{b}}$ & $15.1 \pm 0.7^{\mathrm{c}}$ & $2.9 \pm 1.1^{\mathrm{a}}$ & $5.8 \pm 2.7^{\mathrm{a}}$ & $12.2 \pm 0.8^{\mathrm{b}}$ \\
\hline $15: 0$ & $0.57 \pm 0.30$ & $0.012 \pm 0.002$ & $0.74 \pm 0.08^{\mathrm{a}}$ & $0.62 \pm 0.05^{\mathrm{a}}$ & $0.19 \pm 0.02^{\mathrm{a}}$ & $0.62 \pm 0.14^{\mathrm{a}}$ & $0.61 \pm 0.19^{\mathrm{a}}$ & $0.78 \pm 0.05^{\mathrm{a}}$ \\
\hline $16: 0$ & $21 \pm 2$ & $0.83 \pm 0.19 *$ & $16.8 \pm 1.8^{\mathrm{a}}$ & $9.3 \pm 1.1^{\mathrm{b}}$ & $15.6 \pm 0.4^{\mathrm{a}}$ & $15.1 \pm 1.6^{\mathrm{a}}$ & $10.4 \pm 1.2^{\mathrm{b}}$ & $26 \pm 1^{\mathrm{c}}$ \\
\hline $16: \ln 9$ & $0.06 \pm 0.02$ & $0.006 \pm 0.003$ & $18.9 \pm 1.6^{\mathrm{a}}$ & $20 \pm 2^{\mathrm{a}}$ & $19.1 \pm 0.2^{\mathrm{a}}$ & $0.30 \pm 0.08^{\mathrm{a}}$ & $0.42 \pm 0.16^{\mathrm{a}}$ & $1.62 \pm 0.13^{\mathrm{a}}$ \\
\hline $16: 1 \mathrm{n} 7$ & $0.40 \pm 0.1$ & $0.015 \pm 0.004$ & $5.5 \pm 0.7^{\mathrm{a}}$ & $6.9 \pm 0.8^{\mathrm{a}}$ & $5.7 \pm 0.2^{\mathrm{a}}$ & $3.78 \pm 1.7^{\mathrm{a}}$ & $5.34 \pm 1.9^{\mathrm{a}}$ & $6.35 \pm 0.3^{\mathrm{a}}$ \\
\hline $17: 0$ & $0.80 \pm 0.35$ & $0.014 \pm 0.001$ & $0.79 \pm 0.06^{\mathrm{a}}$ & $0.60 \pm 0.05^{\mathrm{a}}$ & $0.18 \pm 0$ & $0.80 \pm 0.2^{\mathrm{a}}$ & $0.79 \pm 0.3^{\mathrm{a}}$ & $1.75 \pm 0.08^{\mathrm{a}}$ \\
\hline 18:0 & $9 \pm 1.2$ & $0.32 \pm 0.04^{*}$ & $0.5^{\mathrm{a}}$ & $2.0 \pm 0.2^{\mathrm{b}}$ & $2.4 \pm 0.2^{\mathrm{b}}$ & $7.1=$ & $6.2 \pm 0.8^{\mathrm{a}}$ & $9.5 \pm 0.5^{\mathrm{a}}$ \\
\hline $18: 1 n 9$ & $2.9 \pm 1.0$ & $2 *$ & $13.7=$ & $8.3 \pm 1.1^{\mathrm{b}}$ & $14.9 \pm 0.4^{\mathrm{a}}$ & $11.9 \pm 1.7^{\mathrm{a}}$ & $10.1 \pm 1.1^{\mathrm{b}}$ & $15.6 \pm 0.7^{\mathrm{b}}$ \\
\hline $18: 1 \mathrm{n} 7$ & $.28 \pm 0.11$ & $0.039 \pm 0.009$ & $2.2 \pm 0.2^{\mathrm{a}}$ & $0.52 \pm 0.05^{\mathrm{b}}$ & $0.57 \pm 0.09^{\mathrm{a}}$ & $1.96 \pm 0.63^{\mathrm{a}}$ & $1.62 \pm 0.74^{\mathrm{a}}$ & $3.47 \pm 0.08^{\mathrm{a}}$ \\
\hline $18: 2 \mathrm{n} 6$ & $69 \pm 0.09$ & $2.32 \pm 0.5^{*}$ & $11.8 \pm 1.5^{\mathrm{a}}$ & $4.0 \pm 0.3^{\mathrm{b}}$ & $7.8 \pm 0.5^{\mathrm{c}}$ & $8.5 \pm 0.9^{\mathrm{a}}$ & $2.2 \pm 1.0^{\mathrm{b}}$ & $5.6 \pm 0.24^{\mathrm{a}}$ \\
\hline $18: 3 \mathrm{n} 6$ & $<\mathrm{DL}$ & $<\mathrm{DL}$ & $0.19 \pm 0.02^{\mathrm{a}}$ & $<\mathrm{DL}$ & $<\mathrm{DL}$ & $0.20 \pm 0.06^{\mathrm{a}}$ & $0.18 \pm 0.08^{\mathrm{a}}$ & $0.50 \pm 0.07^{\mathrm{a}}$ \\
\hline $18: 3 n 3$ & $<\mathrm{DL}$ & $0.48 \pm 0.11$ & $2.5 \pm 0.3^{\mathrm{a}}$ & $0.34 \pm 0.02^{b}$ & $0.95 \pm 0.06$ & $1.06 \pm 0.2^{\mathrm{a}}$ & $0.17 \pm 0.08^{\mathrm{a}}$ & $1.04 \pm 0.14^{\mathrm{a}}$ \\
\hline $20: 0$ & $42 \pm 0.63$ & $0.033 \pm 0.001 *$ & $0.37 \pm 0.03^{\mathrm{a}}$ & $0.37 \pm 0.04^{\mathrm{a}}$ & $0.10 \pm 0.02^{\mathrm{a}}$ & $0.26 \pm 0.1^{\mathrm{a}}$ & $0.20 \pm 0.1^{\mathrm{a}}$ & $0.06 \pm 0.02^{\mathrm{a}}$ \\
\hline $20: \ln 9$ & $.65 \pm 0.22$ & $0.027 \pm 0.003$ & $1.49 \pm 0.09^{\mathrm{a}}$ & $0.05 \pm 0.02^{\mathrm{a}}$ & $0.44 \pm 0.06^{\mathrm{a}}$ & $0.87 \pm 0.4^{\mathrm{a}}$ & $0.31 \pm 0.24^{\mathrm{a}}$ & $0.22 \pm 0.01^{\mathrm{a}}$ \\
\hline $20: 2 \mathrm{n} 6$ & $<\mathrm{DL}$ & $<\mathrm{DL}$ & $0.29 \pm$ & $<\mathrm{DL}$ & $<\mathrm{DL}$ & $0.28 \pm 0.1^{\mathrm{a}}$ & $0.12 \pm 0.06^{\mathrm{a}}$ & $0.23 \pm 0.03^{\mathrm{a}}$ \\
\hline $20: 3 n 3$ & $<\mathrm{DL}$ & $<\mathrm{DL}$ & $0.21 \pm 0.01^{\mathrm{a}}$ & $<\mathrm{DL}$ & $<\mathrm{DL}$ & $0.12 \pm 0.04^{\mathrm{a}}$ & $0.19 \pm 0.30^{\mathrm{a}}$ & $<\mathrm{DL}$ \\
\hline $20: 3 n 6$ & $<\mathrm{DL}$ & $<\mathrm{DL}$ & $0.20 \pm 0.01^{\mathrm{a}}$ & $<\mathrm{DL}$ & $<\mathrm{DL}$ & $0.47 \pm 0.17^{\mathrm{a}}$ & $0.75 \pm 0.43^{\mathrm{a}}$ & $0.11 \pm 0.03^{\mathrm{a}}$ \\
\hline $20: 4 n 6$ & $<\mathrm{DL}$ & $<\mathrm{DL}$ & $1.20 \pm 0.06^{\mathrm{a}}$ & $0.13 \pm 0.02^{\mathrm{a}}$ & $<\mathrm{DL}$ & $1.85 \pm 0.83^{\mathrm{a}}$ & $1.97 \pm 0.9^{\mathrm{a}}$ & $4.31 \pm 0.3^{\mathrm{a}}$ \\
\hline $20: 5 n 3$ & $<\mathrm{DL}$ & $<\mathrm{DL}$ & $11.8 \pm 1.0^{\mathrm{a}}$ & $0.14 \pm 0.01^{\mathrm{b}}$ & $<\mathrm{DL}$ & $5.30 \pm 0.8^{\mathrm{a}}$ & $0.50 \pm 0.32^{b}$ & $0.58 \pm 0.03^{b}$ \\
\hline $21: 0$ & $0.17 \pm 0.07$ & $<\mathrm{DL}$ & $0.08 \pm 0.01^{\mathrm{a}}$ & $<\mathrm{DL}$ & $<\mathrm{DL}$ & $0.14 \pm 0.07^{\mathrm{a}}$ & $0.11 \pm 0.07^{\mathrm{a}}$ & $0.22 \pm 0.06^{\mathrm{a}}$ \\
\hline $22: 0$ & $2.68 \pm 0.94$ & $0.027 \pm 0.004 *$ & $0.27 \pm 0.03^{\mathrm{a}}$ & $0.48 \pm 0.07^{\mathrm{a}}$ & $<\mathrm{DL}$ & $0.28 \pm 0.22^{\mathrm{a}}$ & $0.25 \pm 0.2^{\mathrm{a}}$ & $<\mathrm{DL}$ \\
\hline $22: 1 n 9$ & $<\mathrm{DL}$ & $<\mathrm{DL}$ & $1.01 \pm 0.05^{\mathrm{a}}$ & $0.015 \pm 0.007^{\mathrm{a}}$ & $<\mathrm{DL}$ & & $0.18 \pm 0.19^{\mathrm{a}}$ & $<\mathrm{DL}$ \\
\hline $22: 6 r$ & $<\mathrm{DL}$ & $<\mathrm{DL}$ & & & $<\mathrm{DL}$ & & $4.7 \pm 0.5^{b}$ & $3.8 \pm 0.4^{b}$ \\
\hline $24: 1 n 9$ & $<\mathrm{DL}$ & $<\mathrm{DL}$ & $0.80 \pm 0.07^{\mathrm{a}}$ & $0.036 \pm 0.008^{\mathrm{a}}$ & $<\mathrm{DL}$ & $0.45 \pm 0.12^{\mathrm{a}}$ & $0.18 \pm 0.17^{\mathrm{a}}$ & $<\mathrm{DL}$ \\
\hline
\end{tabular}

For each matrix, mean within rows bearing different letters are significantly different $(p<0.05)$.

*Indicates statistically significant difference between substrate A and B $(P<0.05)$. 


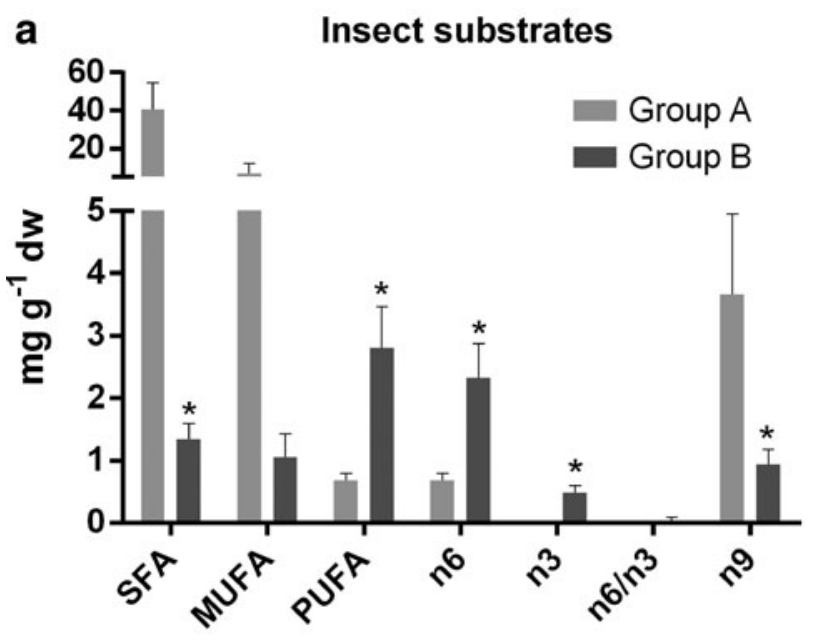

b Experimental diets
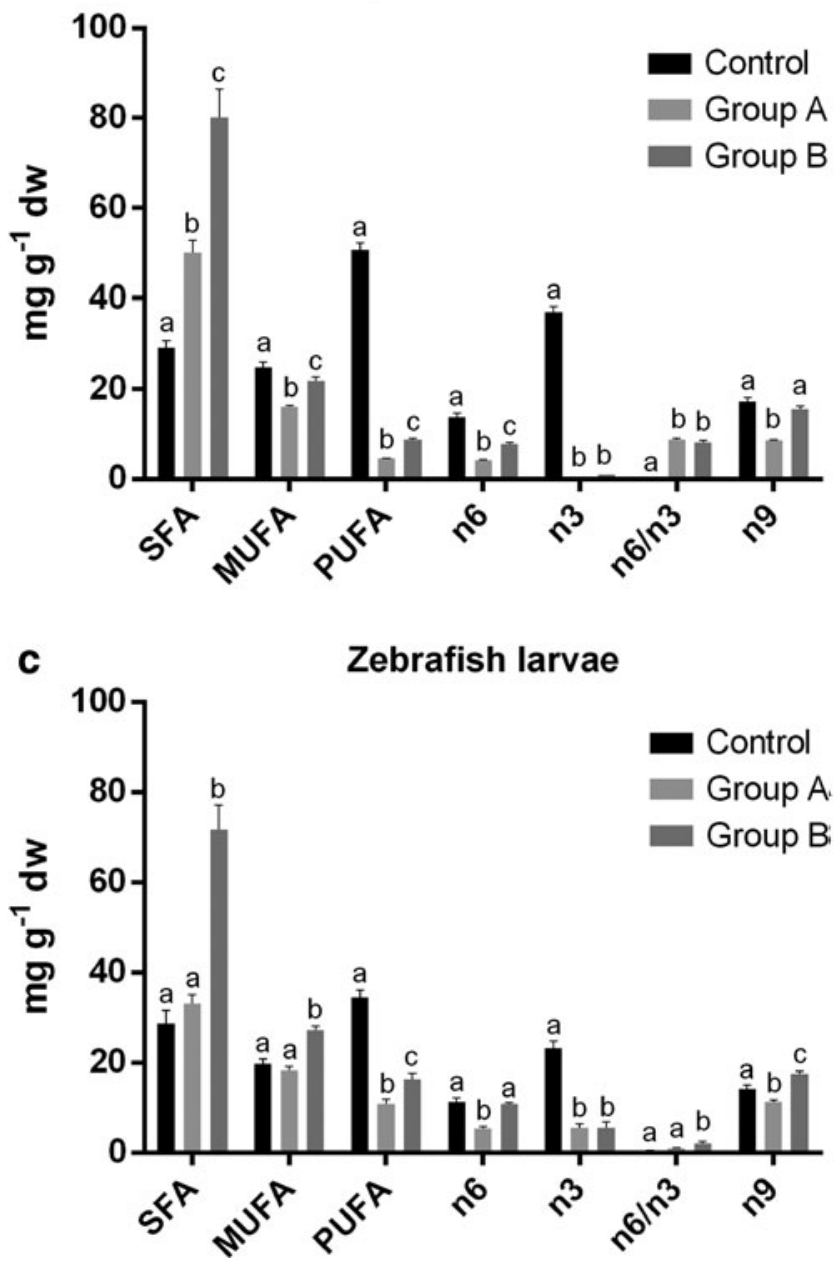

FIG. 1. Composition of SFA, MUFA, and PUFA (as mg $\mathrm{g}^{-1} \mathrm{dw}$ ) in the insect substrates (a), experimental diets, (b) and zebrafish larvae (c), and contribution of omega-3 (n3), omega-6 (n6), and omega-9 (n9) FAs to lipid profile. Different letters indicate statistically significant differences among experimental groups $(p<0.05) ; N=3$. SFA, saturated fatty acid; MUFA, monounsaturated fatty acid; PUFA, polyunsaturated fatty acid.
Considering FA classes, the contribution of n6, n3, and $n 9$ on lipid profile significantly varied among the three diets $(p<0.05)$. The 6 content in group A $\left(4.2 \pm 0.1 \mathrm{mg} \mathrm{g}^{-1} \mathrm{dw}\right)$ and in group $B\left(7.8 \pm 0.3 \mathrm{mg} \mathrm{g}^{-1} \mathrm{dw}\right)$ showed a significantly lower $(p<0.05)$ value with respect to control group $(13.7 \pm 0.9 \mathrm{mg}$ $\left.\mathrm{g}^{-1} \mathrm{dw}\right)$. The $\mathrm{n} 3$ content in both group A $\left(0.50 \pm 0.01 \mathrm{mg} \mathrm{g}^{-1}\right.$ $\mathrm{dw})$ and group $\mathrm{B}\left(1.00 \pm 0.03 \mathrm{mg} \mathrm{g}^{-1} \mathrm{dw}\right)$ was significantly $(p<0.05)$ lower with respect to control group $(37.1 \pm 1.2 \mathrm{mg}$ $\left.\mathrm{g}^{-1} \mathrm{dw}\right)$. Consequently, the $\mathrm{n} 6 / \mathrm{n} 3$ ratio was significantly higher $(p<0.05)$ in both group A $\left(8.8 \pm 0.3 \mathrm{mg} \mathrm{g}^{-1} \mathrm{dw}\right)$ and group B $\left(8.2 \pm 0.4 \mathrm{mg} \mathrm{g}^{-1} \mathrm{dw}\right)$ with respect to control group $(0.37 \pm$ $\left.0.03 \mathrm{mg} \mathrm{g}^{-1} \mathrm{dw}\right)$ diet. Finally, the n9 FA content in group A diet $\left(8.6 \pm 0.2 \mathrm{mg} \mathrm{g}^{-1} \mathrm{dw}\right)$ was significantly lower $(p<0.05)$ with respect to both control group $\left(17.2 \pm 1.0 \mathrm{mg} \mathrm{g}^{-1} \mathrm{dw}\right)$ and group B (15.6 $\left.\pm 0.6 \mathrm{mg} \mathrm{g}^{-1} \mathrm{dw}\right)$ diets.

Zebrafish larvae. Group A did not show significant $(p>0.05)$ differences in SFA $\left(33 \pm 2 \mathrm{mg} \mathrm{g}^{-1} \mathrm{dw}\right)$ and MUFA content $\left(18.2 \pm 0.9 \mathrm{mg} \mathrm{g}^{-1} \mathrm{dw}\right)$ with respect to control group (SFA 28.7 $\pm 2.8 \mathrm{mg} \mathrm{g}^{-1} \mathrm{dw}$ and MUFA $19.8 \pm 1.1 \mathrm{mg} \mathrm{g}^{-1} \mathrm{dw}$ ), whereas group B showed a significantly higher content (SFA $71.8 \pm 5.4 \mathrm{mg} \mathrm{g}^{-1} \mathrm{dw}$ and MUFA $27.2 \pm 0.8 \mathrm{mg} \mathrm{g}^{-1} \mathrm{dw} ; p<$ 0.05 ) with respect to both control group and group A (Fig. 1c). Regarding PUFAs, both group A and B larvae showed a significantly lower $(p<0.05)$ content $(10.8 \pm 1.1$ and $16.2 \pm$ $1.5 \mathrm{mg} \mathrm{g}^{-1} \mathrm{dw}$, respectively) with respect to control group $\left(34.5 \pm 1.6 \mathrm{mg} \mathrm{g}^{-1} \mathrm{dw}\right)$; group A showed significantly lower $(p<0.05)$ PUFA content with respect to group B.

Considering the n6 content, group A larvae showed a significantly lower $(p<0.05) \mathrm{n} 6$ content $\left(5.3 \pm 0.6 \mathrm{mg} \mathrm{g}^{-1} \mathrm{dw}\right)$ with respect to both control group and group B (11.3 \pm 0.8 and $10.8 \pm 0.4 \mathrm{mg} \mathrm{g}^{-1} \mathrm{dw}$, respectively). As regards the $\mathrm{n} 3$ content, both group A and B larvae showed a significantly lower $(p<0.05) \mathrm{n} 3$ content $\left(5.5 \pm 0.9 \mathrm{mg} \mathrm{g}^{-1} \mathrm{dw}\right.$ and $5.4 \pm 1.4 \mathrm{mg} \mathrm{g}^{-1}$ $\mathrm{dw}$, respectively) with respect to control group $(23.2 \pm 1.5$ $\mathrm{mg} \mathrm{g}^{-1} \mathrm{dw}$; Fig. 1c). Consequently, the $\mathrm{n} 6 / \mathrm{n} 3$ ratio in group A $\left(1.0 \pm 0.1 \mathrm{mg} \mathrm{g}^{-1} \mathrm{dw}\right)$ did not show significant differences $(p>0.05)$ with respect to control group $\left(0.5 \pm 0.04 \mathrm{mg} \mathrm{g}^{-1} \mathrm{dw}\right)$, whereas group B $\left(2.0 \pm 0.5 \mathrm{mg} \mathrm{g}^{-1} \mathrm{dw}\right)$ showed a significantly higher value $(p<0.05)$ with respect to both group $\mathrm{A}$ and control group. Finally, as regards the $n 9$ content, group A $\left(11.3 \pm 0.5 \mathrm{mg} \mathrm{g}^{-1} \mathrm{dw}\right)$ showed significantly lower values $(p<0.05)$ with respect to control group $\left(14.1 \pm 0.9 \mathrm{mg} \mathrm{g}^{-1}\right.$ $\mathrm{dw})$, whereas group B $\left(17.4 \pm 0.7 \mathrm{mg} \mathrm{g}^{-1} \mathrm{dw}\right)$ showed a significantly higher $(p<0.05)$ value with respect to both control group and group A.

\section{Histological and infrared analysis}

No appreciable differences among larvae fed BSF diets (group A and group B) and a commercial feed (control group) were detected at 7 and $14 \mathrm{dps}$ in the intestinal mucosa of all analyzed samples (Fig. 2a-f). A general increase in goblet cell number was evident at $21 \mathrm{dps}$ only in group B samples with respect to both control group and group A (Fig. 2 g-i). In addition, no severe histological alterations were thus observed in all experimental groups in terms of inflammatory influx and morphological alterations of intestinal folds.

For a deeper insight of the aforementioned modifications, the FTIRM analysis of intestine sections from 21 dps zebrafish larvae fed BSF meals (group A and group B) and a commercial meal (control group) was performed. By the 


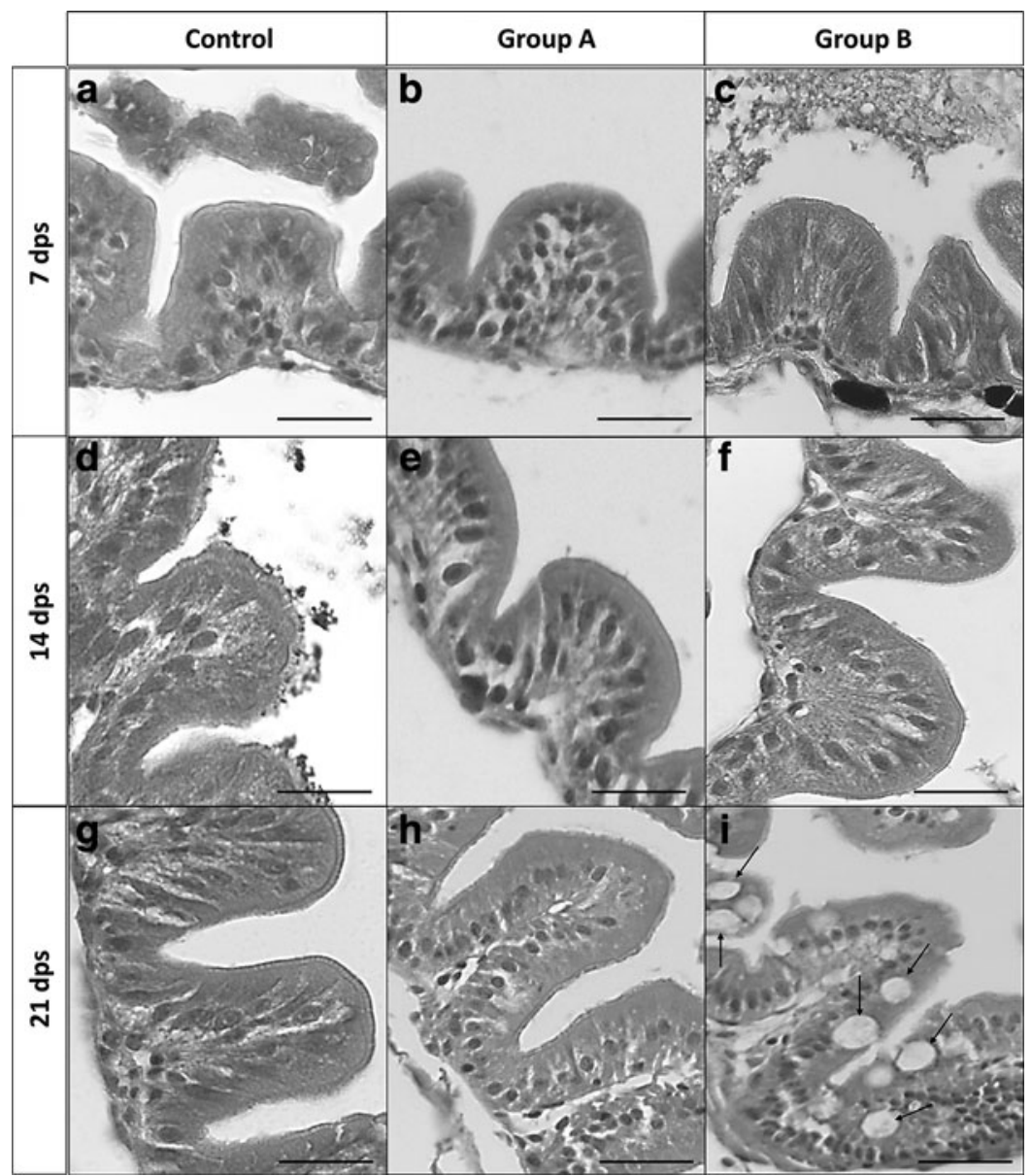

FIG. 2. Intestine morphology (E\&E) of zebrafish larvae fed two different BSF diets (group A and group B) and a commercial feed (control) collected at 7, 14, and $21 \mathrm{dps}$ (group A: b, e, h; group B: c, f, i; control: a, d, g). Group A: zebrafish larvae fed BSF grown on byproducts obtained from roasting coffee process; group B: zebrafish larvae fed BSF grown on corn meal, and fruit and vegetable mixture (50:50). A general increase in goblet cell number is evident at $21 \mathrm{dps}$ in group B samples (i, arrows). Scale bars: $25 \mu \mathrm{m}$. BSF, black soldier fly. analysis of the IR spectra of each experimental group, the following absorption bands were detected: $3016 \mathrm{~cm}^{-1}(=\mathrm{CH}$ stretching mode of lipid alkyl chains $)^{42} ; 2960,2873 \mathrm{~cm}^{-1}$ $\left(\mathrm{CH}_{3}\right.$ asymmetric and symmetric stretching modes of lipid alkyl chains, $v_{\text {asym }} \mathrm{CH}_{3}$ and $v_{\mathrm{sym}} \mathrm{CH}_{3}$ ), and $2926,2850 \mathrm{~cm}^{-1}$ $\left(\mathrm{CH}_{2}\right.$ asymmetric and symmetric stretching modes of lipid alkyl chains, $v$ asym $\mathrm{CH}_{2}$ and $\left.v_{\text {sym }} \mathrm{CH}_{2}\right)^{42} ; 1747 \mathrm{~cm}^{-1}$ (vibrational modes of fatty acids) ${ }^{43} ; 1653$ and $1546 \mathrm{~cm}^{-1}$ (Amide I and Amide II bands of proteins, attributable to $\mathrm{C}=\mathrm{O}$ and $\mathrm{C}-\mathrm{N}$ stretching, and N-H bending modes $)^{44} ; 1455 \mathrm{~cm}^{-1}\left(\mathrm{CH}_{2}\right.$ bending modes of alkyl chains) ${ }^{45} ; 1399 \mathrm{~cm}^{-1}$ (COO- carboxylate groups of aspartate and glutamate amino acids $)^{45,46}$; $1241 \mathrm{~cm}^{-1}$ (collagen vibrational modes) ${ }^{47} ; 1143 \mathrm{~cm}^{-1}(\mathrm{C}-\mathrm{OH}$ stretching vibrations of carbohydrates) ${ }^{45}$; and 1100 and $1040 \mathrm{~cm}^{-1}$ (glycosylated proteins). ${ }^{48}$

A semiquantitative analysis was performed on the spectra extracted from IR maps. For this purpose, the following band area ratios were calculated: LIP/TBM (representative of total lipids); FA/TBM (representative of total fatty acids); $\mathrm{CH} 2$ / TBM (representative of alkyl chain length); PRT/TBM (representative of total proteins), and MUCIN/TBM (representative of total mucin) (Fig. 3a-e). Their variation among the experimental groups was analyzed. In group A and group $\mathrm{B}$, a statistically significant increase $(p<0.05)$ of lipids (LIP/ TBM, $0.24 \pm 0.02$ and $0.38 \pm 0.02$, respectively; Fig. $3 e$ ) and in particular of fatty acids (FA/TBM, $0.20 \pm 0.10$ and $0.25 \pm 0.05$; Fig. $3 b$ ) with longer alkyl chains (CH2/TBM2, $0.04 \pm 0.00$ and $0.07 \pm 0.01$; Fig. $3 c$ ) was observed with re- spect to control group (LIP/TBM $0.08 \pm 0.02$, FA/TBM $0.07 \pm 0.01$ and $\mathrm{CH} 2 / \mathrm{TBM} 0.02 \pm 0.01$; Fig. 3a-c). Similarly, a significantly higher $(p<0.05)$ proportion of proteins (PRT/ TBM) was also detected in group A $(0.96 \pm 0.11)$ and group B $(1.04 \pm 0.15)$ with respect to control group $(0.71 \pm 0.09$; Fig. 3a), above all attributable to mucin (MUCIN/TBM, group A $0.66 \pm 0.10$, group B $0.76 \pm 0.09$ and control group $0.19 \pm 0.05$; Fig. 3d).

Concerning the histological analysis of the liver, group A samples did not show any alteration at the hepatocyte level during the whole experimental period (Fig. 4b, e, h), with a general aspect comparable to the control group (Fig. 4a, d, g). In group B samples, no morphological alterations were observed with respect to control group until 7dps (Fig. 4c); however, at 14 and $21 \mathrm{dps,}$ a general hepatic steatosis, with a conspicuous intracellular accumulation of lipids, was observed (Fig. 4f, i).

\section{Real-time $P C R$ results}

Real-time PCR analysis was performed on genes involved in fish growth (igfl, igf $2 a$, and $m s t n b)$, fatty acid desaturation (fad2) and elongation (elovl5 and elovl2), stress response ( $n r 3 c l$ and $h s p 70.1$ ), and enzymatic hydrolysis of chitin (chia.1, chia.2, chia.3, chia.4, chia.5, and chia.6).

Growth factors. Regarding igfl, a time-dependent increase in gene expression was evident in all experimental groups, with no significant differences $(p>0.05)$ among 

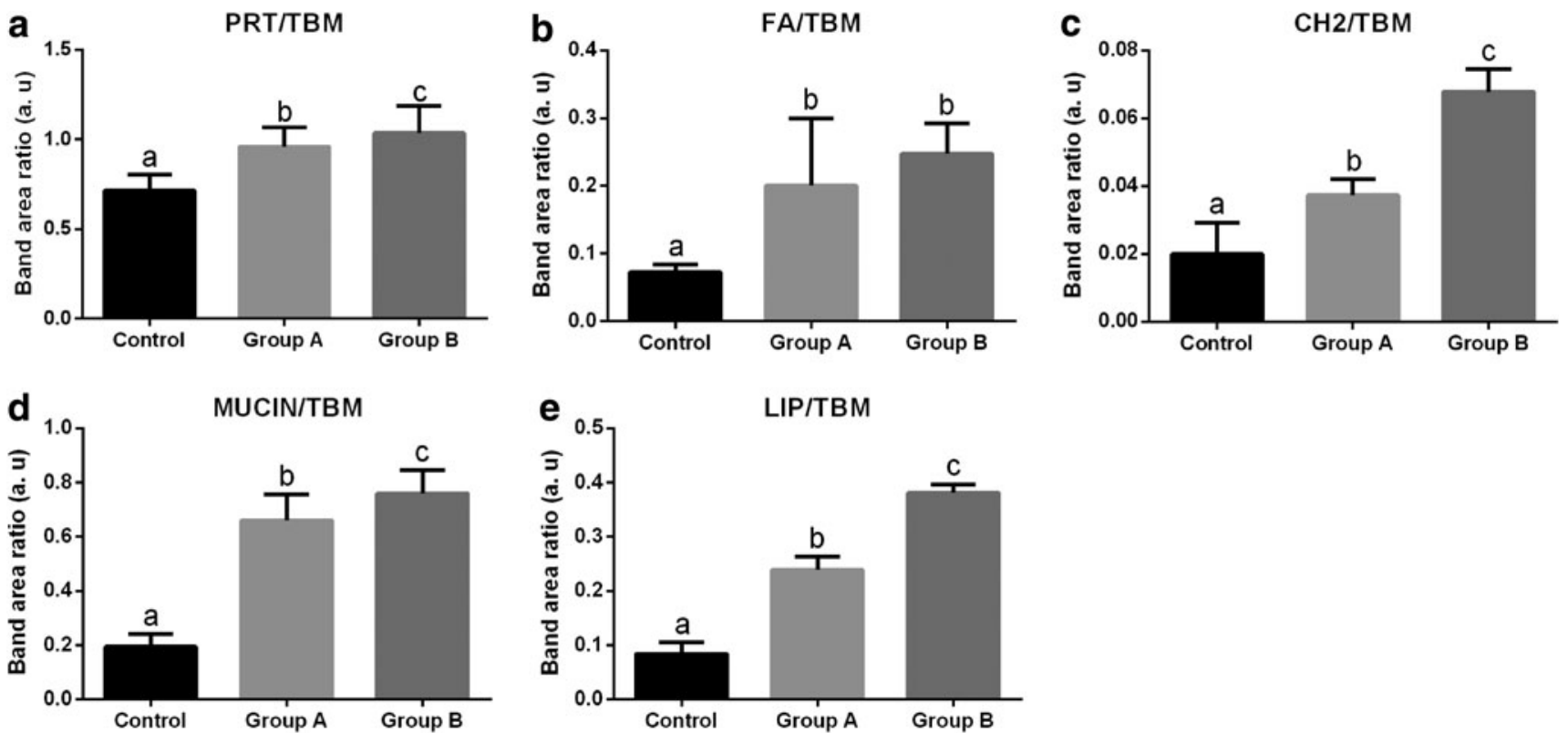

FIG. 3. Numerical variations of the following band area ratios calculated on IR spectra of intestine sections from $21 \mathrm{dps}$ zebrafish larvae fed two different BSF diets (group A and group B) and a commercial feed (control group): LIP/TBM, FA/ TBM, CH2/TBM, PRT/TBM, and MUCIN/TBM (a, b, c, d, e respectively). Different letters indicate statistically significant differences among experimental groups $(p<0.05)$.

them (Fig. 5a). A similar result was observed for igf $2 a$ gene expression, with the exception of group B samples, which showed a significantly lower $(p<0.05)$ gene expression at $14 \mathrm{dps}$ and a significantly higher $(p<0.05)$ gene expression at $21 \mathrm{dps}$ with respect to both control group and group A
(Fig. 5b). Finally, as regards mstnb gene expression, results did not show a similar trend to igfs. Particularly, group A samples did not show significant differences $(p>0.05)$ with respect to control group at 7 and $14 \mathrm{dps,} \mathrm{whereas} \mathrm{a} \mathrm{significant}$ downregulation $(p<0.05)$ was observed at $21 \mathrm{dps}$ (Fig. 5c).

FIG. 4. Liver morphology (E\&E) of zebrafish larvae fed two different BSF diets (group A and group B) and a commercial feed (control) collected at 7, 14, and $21 \mathrm{dps}$. Group A: zebrafish larvae fed BSF grown on byproducts obtained from roasting coffee process; group B: zebrafish larvae fed BSF grown on corn meal, and fruit and vegetable mixture (50:50). At $7 \mathrm{dps,} \mathrm{no} \mathrm{histological}$ alterations were observed in all experimental groups (a-c). No histological alterations were observed in $(\mathbf{d}, \mathbf{g})$ and in group $\mathrm{A}(\mathbf{e}, \mathbf{h})$ at 14 and $21 \mathrm{dps}$. A general hepatic steatosis is evident at 14 and $21 \mathrm{dps}$ in group (B) (f, i). Scale bars: $5 \mu \mathrm{m}$.

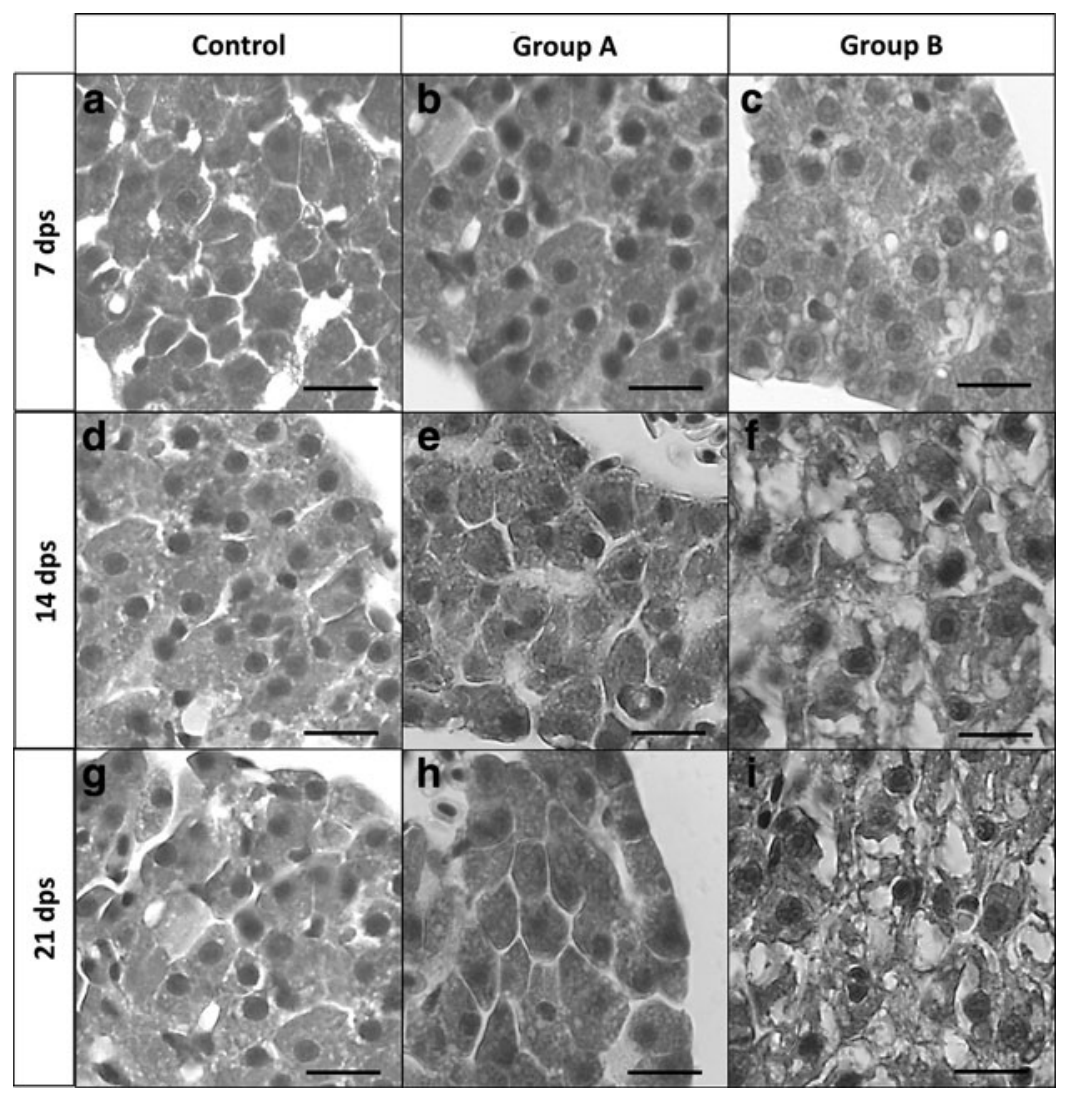



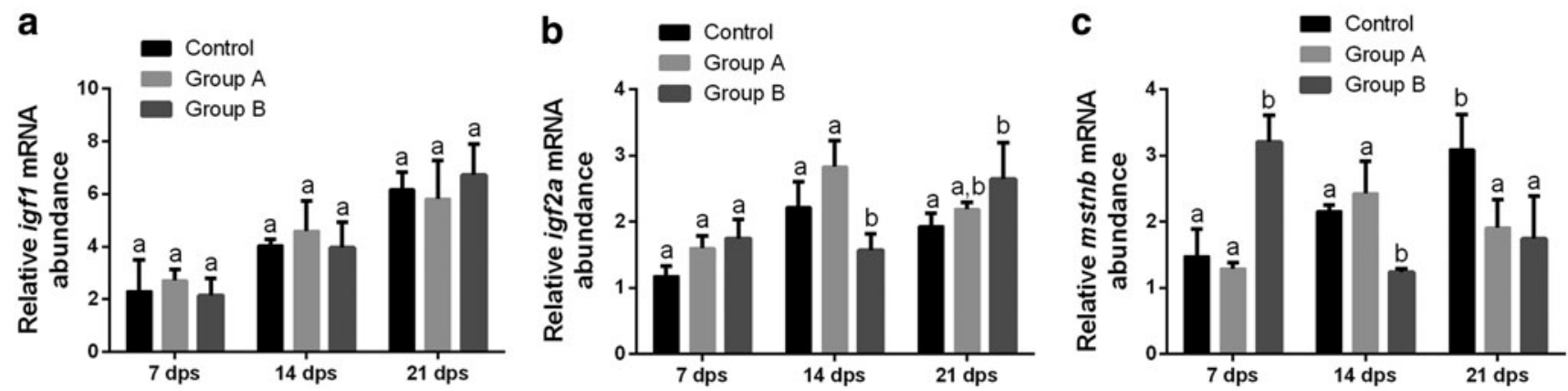

FIG. 5. Relative mRNA abundance of genes involved in fish growth (igfl, igf $2 a$, and $m s t n b$, a-c respectively) analyzed in zebrafish pools of 20 larvae, fed two different BSF diets group A and B and a commercial feed (control) collected at 7, 14, and 21 days postspawning (dps). Group A: zebrafish larvae fed BSF grown on byproducts obtained from roasting coffee process; group B: zebrafish larvae fed BSF grown on corn meal, and fruit and vegetable mixture (50:50). Different letters indicate statistically significant differences among experimental groups compared at the same experimental time $(p<0.05) ; N=3$.

Group B samples showed a significantly higher $(p<0.05)$ $m s t n b$ gene expression at $7 \mathrm{dps}$ with respect to both control group and group $\mathrm{A}$, whereas, at $14 \mathrm{dps}$, a significant downregulation $(p<0.05)$ in its gene expression was observed. Finally, at $21 \mathrm{dps}$, a significantly lower $(p<0.05)$ gene expression with respect only to control group was evidenced.

Markers of long-chain polyunsaturated fatty acid biosynthesis. As regards the genes involved in the long-chain polyunsaturated fatty acid biosynthesis, results showed that all desaturases and elongases, in both group A and B, were principally expressed at $21 \mathrm{dps}$. Particularly, as mentioned above, fad 2 gene expression, in both group A and B samples did not show significant differences $(p>0.05)$ with respect to control group at 7 and $14 \mathrm{dps}$, whereas at $21 \mathrm{dps}$ both group A and B showed a significant upregulation $(p<0.05)$ with respect to control group (Fig. 6a). A similar result was observed for elovl5 gene expression (Fig. 6b). Finally, as concerns elovl2 gene expression, group A samples did not show significant differences $(p>0.05)$ with respect to control group at 7 and $14 \mathrm{dps}$, whereas at $21 \mathrm{dps}$ a significant upregulation $(p<0.05)$ with respect to control group was evident (Fig. 6c). Group B samples, at $7 \mathrm{dps}$, did not show any significant difference $(p>0.05)$ in its elovl2 gene expression with respect to control group, whereas at $14 \mathrm{dps}$ a significantly higher $(p<0.05)$ gene expression in group B samples with respect to group A was observed (Fig. 6c). Finally, at $21 \mathrm{dps}$, a significantly higher $(p<0.05)$ gene expression was detected only with respect to control group, whereas a significantly lower $(p<0.05)$ gene expression was observed with respect to group A (Fig. 6c).

Stress markers. As regards $n r 3 c l$ gene expression, group A samples did not show significant differences $(p>0.05)$ with respect to control group during the whole experimental period (Fig. 7a). On the contrary, group B samples showed a significantly higher $(p<0.05)$ gene expression only at $7 \mathrm{dps}$ with respect to both control group and group A. Finally, at $21 \mathrm{dps}$, a significantly lower $(p<0.05)$ gene expression in group B samples, only with respect to group A, was detected.

As concerns $h s p 70.1$ gene expression, group A samples did not show any significant difference $(p>0.05)$ with respect to control group during the whole experimental period (Fig. 7b). Group B samples showed a significantly lower $(p<0.05)$ gene expression at $7 \mathrm{dps}$ with respect to both control group and group A, whereas at $14 \mathrm{dps}$ a significantly higher $(p<0.05)$ gene expression was observed. Finally, at $21 \mathrm{dps}$ no significant differences $(p>0.05)$ among experimental groups were observed.

Markers of enzymatic hydrolysis of chitin. As concerns the enzymatic hydrolysis of chitin, in general, all six genes
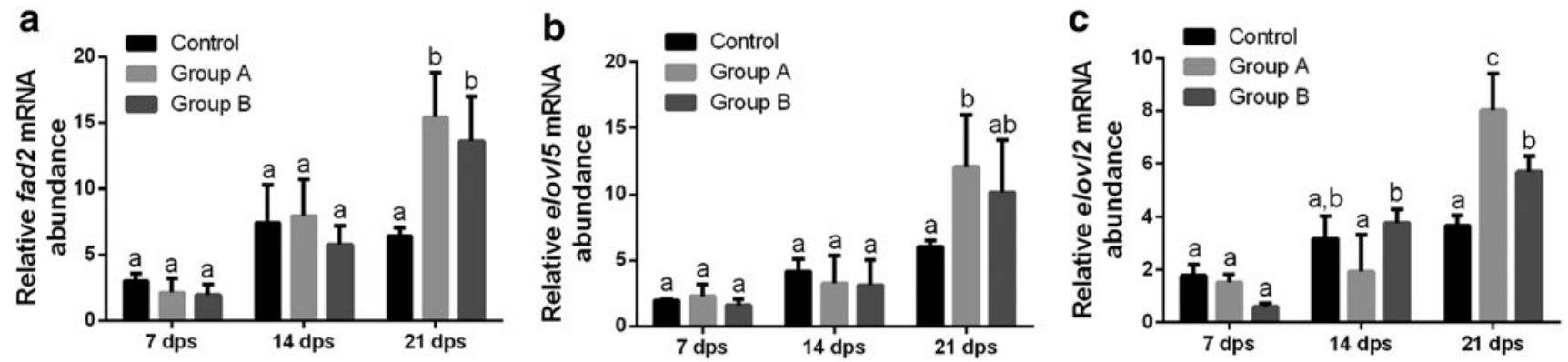

FIG. 6. Relative mRNA abundance of genes involved in long-chain polyunsaturated fatty acid biosynthesis (fad2, elovl5, and elovl2, a-c respectively) analyzed in zebrafish pools of 20 larvae, fed two different BSF diets group A and B and a commercial feed (control) collected at 7, 14, and 21 days postspawning (dps). Group A: zebrafish larvae fed BSF grown on byproducts obtained from roasting coffee process; group B: zebrafish larvae fed BSF grown on corn meal, and fruit and vegetable mixture (50:50). Different letters indicate statistically significant differences among experimental groups compared at the same experimental time $(p<0.05) ; N=3$. 

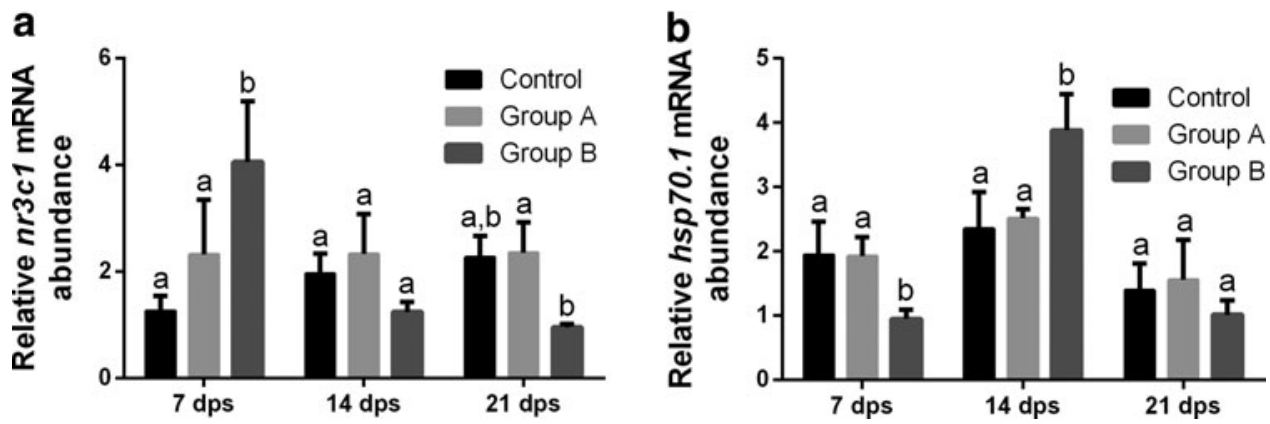

FIG. 7. Relative mRNA abundance of genes involved in stress response ( $n r 3 c 1$ and $h s p 70.1$, a, b respectively) analyzed in zebrafish pools of 20 larvae, fed two different BSF diets group A and B and a commercial feed (control) collected at 7, 14, and 21 days postspawning (dps). Group A: zebrafish larvae fed BSF grown on byproducts obtained from roasting coffee process; group B: zebrafish larvae fed BSF grown on corn meal, and fruit and vegetable mixture (50:50). Different letters indicate statistically significant differences among experimental groups compared at the same experimental time $(p<0.05) ; N=3$.

analyzed showed a time-dependent increase (Fig. 8). Particularly, chia.1,2,3,4,5, and 6, gene expression in group A samples did not show any significant difference $(p>0.05)$ during the whole experimental period with respect to control group, except for chia. 4 at $21 \mathrm{dps}$, at which a significantly lower $(p<0.05)$ gene expression was detected (Fig. 8a-f). Considering group B samples, a more heterogeneous trend was detected with respect to group A.

Particularly, as regards chia.1, no significant differences $(p>0.05)$ at $7 \mathrm{dps}$ among experimental groups were detected, whereas at 14 dps a significantly higher $(p<0.05)$ gene expression, with respect to both control group and group A, was observed in group B (Fig. 8a). Finally at $21 \mathrm{dps,} \mathrm{group} \mathrm{B} \mathrm{did}$ not show any significant difference $(p>0.05)$ among experimental groups (Fig. 8a). As regards chia.2 gene expression, group B samples showed a significantly lower $(p<0.05)$ gene expression at $7 \mathrm{dps}$ with respect to both control group and group A, whereas at $14 \mathrm{dps}$ no significant differences ( $p>0.05)$ among experimental groups were observed (Fig. 8b). Finally at $21 \mathrm{dps}$, group B samples showed a significantly higher $(p<0.05)$ gene expression only with respect to control group. As regards chia.3 and chia.4 gene expression, no significant differences $(p>0.05)$ among experimental groups during the whole experimental period were observed, with the exception of group A samples, which showed a significantly lower $(p<0.05)$ chia. 4 gene expression at $21 \mathrm{dps}$ with respect to control group (Fig. 8c, d). As concerns chia.5 gene expression, group B samples showed a significantly higher $(p<0.05)$ gene expression at $7 \mathrm{dps}$ with respect to both control group and group $\mathrm{A}$, whereas at $14 \mathrm{dps}$ no significant differences $(p>0.05)$ among experimental groups were observed (Fig. 8e). Finally at $21 \mathrm{dps}$, a significantly lower $(p<0.05)$ gene expression in group B samples, with respect to the other experimental groups, was observed (Fig. 8e). As regards chia.6 gene expression, Group B samples showed a significantly lower $(p<0.05)$ gene expression at $7 \mathrm{dps}$ only with respect to

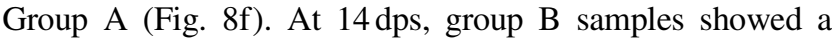
significantly higher $(p<0.05)$ gene expression with respect to control group, whereas at $21 \mathrm{dps}$ no significant differences $(p>0.05)$ among experimental groups were observed.

\section{Discussion}

A desirable goal of the aquaculture sector is to replace most of fish meal and fish oil with more sustainable, cost- effective, and environmentally friendly ingredients. The search for these alternatives represents a major challenge, since alternatives must ensure fish health and welfare standards by providing proper levels of essential amino acids and PUFAs, high nutrient and energy bioavailability, and reduced antinutritional factors. ${ }^{4,6,7}$ Due to minimal environmental impact, compared with most conventional feed commodities, insects deserve a growing attention as candidate ingredients in aquafeeds. ${ }^{10,14,49}$ Insect meal meets macronutrient requirements of many terrestrial animals and fish; former studies showed suitability of this novel ingredient in diets for swine and broiler chickens as well as in nutrition of rainbow trout (Oncorhynchus mykiss), or in channel catfish (Ictalurus punctatus), and blue tilapia (Tilapia aurea). ${ }^{18,50-54}$

Even though zebrafish is a widely used model organism, information about its dietary preferences is limited and its nutritional requirements are mostly unknown. ${ }^{55}$ Wild zebrafish are known to feed on a wide variety of benthic and planktonic crustaceans, worms, and insect larvae. ${ }^{56}$ McClure and collaborators ${ }^{57}$ and Spence and collaborators ${ }^{58}$ analyzed gut contents of zebrafish sampled in the wild and found that insects, mostly of terrestrial origin, were the predominant prey. Information obtained through the present study may thus be used to generalize how several biological processes occur in related organisms, and contribute toward improving our understanding of the mechanisms involved in fish nutrition and growth.

Presently, most of the studies performed on fish meal and fish oil replacement with insect meal in aquaculture, used levels of inclusion between $10 \%$ and $50 \% .^{21,59,60}$ In this regard, the present study investigated, for the first time, the possible application of a $100 \%$ insect diet in zebrafish larval rearing. In this sense, the economic advantage of the present feeds is recognizable, however, the main scope of the present study was to analyze, through a multidisciplinary approach, the major biological responses of fish to the new diets.

Generally, growth and survival results obtained in the present study demonstrated that fish were not significantly impaired by the new diets. These data were fully supported by the molecular results about growth factors (igfs and msntb). However, it should be pointed out that different results have been reported for other fish species. For example, Gasco and collaborators $^{59}$ reported a reduction in growth in seabream fed increasing amounts of Tenebrio molitor $(0.5,1.0 \mathrm{~g} / \mathrm{kg})$, while Lock and collaborators ${ }^{61}$ did not observe negative 

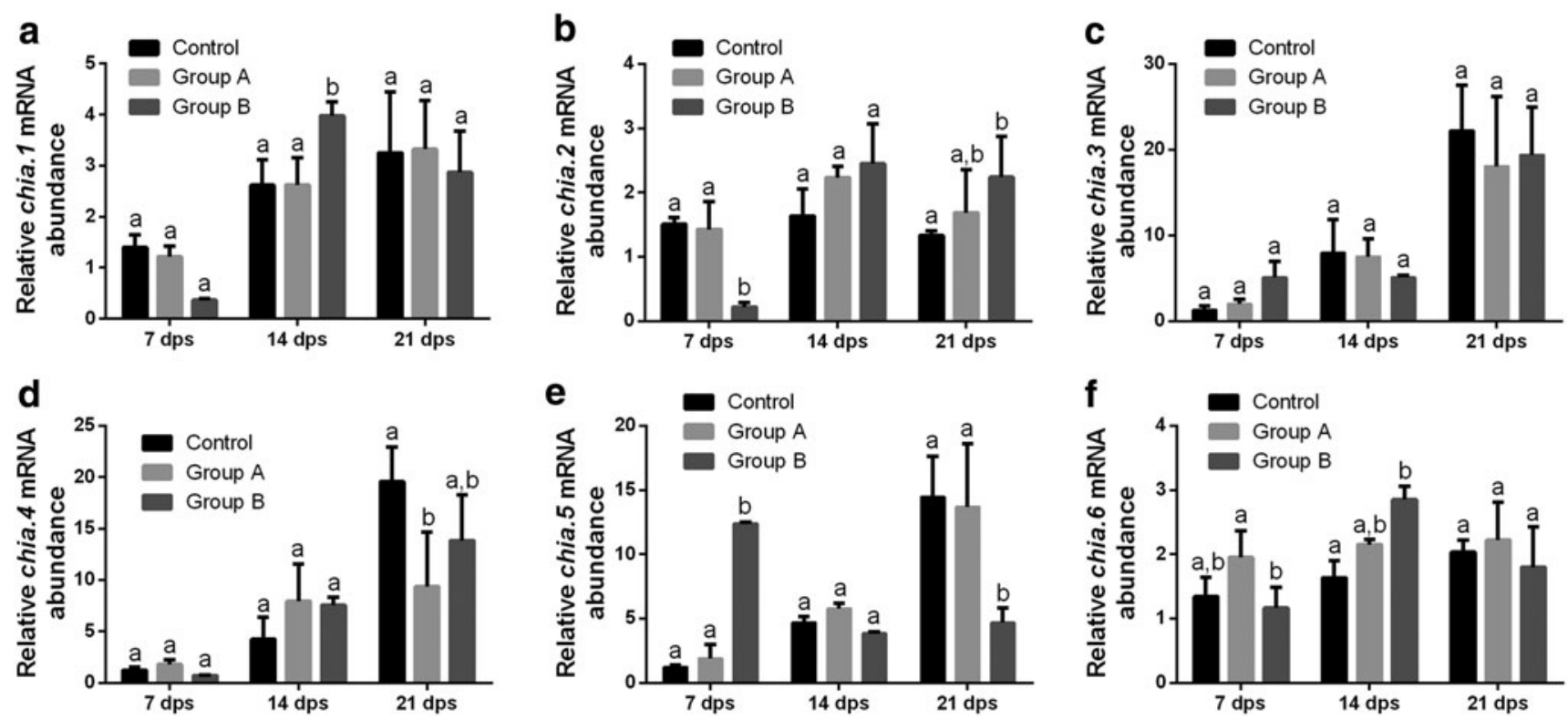

FIG. 8. Relative mRNA abundance of genes involved in enzymatic hydrolysis of chitin (chia. 1, chia.2, chia.3, chia.4, chia.5, and chia.6, a-f respectively) analyzed in zebrafish pools of 20 larvae, fed two different BSF diets group A and B and a commercial feed (control) collected at 7, 14, and 21 days postspawning (dps). Group A: zebrafish larvae fed BSF grown on byproducts obtained from roasting coffee process; group B: zebrafish larvae fed BSF grown on corn meal, and fruit and vegetable mixture (50:50). Different letters indicate statistically significant differences among experimental groups compared at the same experimental time $(p<0.05) ; N=3$.

effects in replacing fish meal with BSF meal in Salmo salar. Clearly, results may be dependent on the insect and fish species used, as well as on the fish developmental stage and on the insect nutritional profile.

As one of the major nutrients, dietary lipids play important roles in fish nutrition. In this regard, it is well established that lipids play a pivotal role in fish larval nutrition and are involved as energy source, component of cellular membranes, and absorption of lipophilic nutrients. ${ }^{62}$ One important aspect of larval fish nutrition is providing adequate levels of highly unsaturated fatty acids (HUFAs), including EPA and $\mathrm{DHA}^{63}$; however, insects are known to be poor in n-3 fatty acids. ${ }^{16,18,64,65}$

In the present study, a high SFA content was observed in both insect diets. Group A and B diets showed a significantly higher SFA content $\left(50.1 \pm 2.8\right.$ and $80.2 \pm 6.2 \mathrm{mg} \mathrm{g}^{-1} \mathrm{dw}$, respectively) and a lower PUFA content $\left(4.6 \pm 0.1\right.$ and $8.7 \pm 0.3 \mathrm{mg} \mathrm{g}^{-1} \mathrm{dw}$, respectively) with respect to control group diet (SFA 29.3 \pm $1.4 \mathrm{mg} \mathrm{g}^{-1} \mathrm{dw}$, PUFA $50.8 \pm 1.5 \mathrm{mg} \mathrm{g}^{-1} \mathrm{dw}$ ), and they were able to affect fish fatty acid composition.

In fish, it is known that a HUFA and/or PUFA deficiency may stimulate an adaptation mechanism by facilitating a level of bioconversion of C18 to C20 or even to C22 FAs. ${ }^{66,67}$ Thus, the differences in the composition of one FA between diets and zebrafish larvae fed these diets could reflect an adaptive mechanism of the fish to equilibrate their FA profile. ${ }^{68}$ Zebrafish larvae were able to synthesize longer chain fatty acids starting from shorter precursors; these data are supported by the higher desaturase and elongase gene expression observed in group A and B with respect to control group at the end of the experiment, and by the GM results.

As already reported, the largest lipid fraction of the insect larvae used in the present study was SFA, of which the majority was represented by the medium-chain fatty acid 12:0 (lauric acid) (27 \pm 5 and $44 \pm 4 \mathrm{mg} \mathrm{g}^{-1} \mathrm{dw}$, group A and B, respectively), which is in line with earlier reports. ${ }^{69}$ Particularly, lauric acid, has been reported as a proper energy source for both humans and livestock, as it is efficiently absorbed, digested, and $\beta$-oxidized (as reviewed by Dayrit ${ }^{70}$ ). Medium-chain fatty acids, mostly $\mathrm{C}_{6}-\mathrm{C}_{12}$, are considered physiologically active compounds, which appear to be preferentially utilized as an energy source and reduce the deposition of adipose tissue. ${ }^{71,72}$ Senlin and collaborators ${ }^{73}$ reported no effects on lipid accumulation in the hepatopancreas and muscle of Jian carp fed insect diets. Similar results have been reported in red drum by Davis and collaborators. ${ }^{74}$ However, in the present study, group B larvae showed, at the end of the experiment, hepatic steatosis that was not observed in the other groups. Differences in liver lipid stores among the present and previous studies might be the result of differences in the fish size/species and/or the duration of relevant feeding experiments. ${ }^{75}$ In addition, group B larvae were fed the fattest diet and this may have a direct effect on lipid steatosis since the amount of lipids provided through the diet was outside the physiologically tolerable range for zebrafish. ${ }^{76}$ This observation is in accordance with FTIRM data, which evidenced a higher amount of lipids in A and B groups at the intestinal level.

The FTIRM analysis of the intestinal tract of $21 \mathrm{dps} z e b-$ rafish larvae fed BSF meals (group A and group B), evidenced a significant increase of mucin (MUCIN/TBM; $0.660 \pm 0.097$ and $0.759 \pm 0.086$, respectively) with respect to control group $(0.195 \pm 0.045)$. A small increment of total proteins (PRT/TBM) was also observed $(0.960 \pm 0.105$ and $1.036 \pm 0.150$, respectively). These spectroscopic findings were in line with the histological analysis, which evidenced in group B a higher number of mucous cells at the intestinal level. These same cells have previously been associated to 
high lipid diets in mice. ${ }^{77,78}$ In addition to compromising metabolic health, ${ }^{76}$ highly saturated fat diets are known to induce low-grade intestinal inflammation. ${ }^{79}$ Besides morphological damages, one of the strategies used to analyze adverse effects on intestine is the evaluation of the number of goblet cells, and this is often used as a gold standard for the evaluation of the intestinal inflammation degree. ${ }^{80}$ In fact, mucous is able to respond to intestine inflammation by protecting the underlined epithelium from exogenous stress. Even if no serious tissue damages were induced in the intestine by diet B, a higher degree of mucous cells was observed and considered as a mild inflammatory response to this diet. Finally, as expected, the fatty acid profiles of the larvae, analyzed in the present study, generally followed the fatty acid profile of the provided diet. In particular, in $21 \mathrm{dps}$ zebrafish larvae fed BSF meals (group A and group B), FTIRM analysis evidenced an increase in SFA: in fact, the increase of FA/TBM (0.200 \pm 0.099 and $0.247 \pm 0.045$, respectively) and $\mathrm{CH} 2 / \mathrm{TBM}(0.0372 \pm 0.0047$ and $0.0678 \pm 0.0067$, respectively) band area ratios suggested a higher amount of fatty acids with longer saturated alkyl chains.

In addition, medium-chain fatty acids have also been reported to possess antibacterial and antiviral properties and have demonstrated positive effects on gut health. ${ }^{81,82} \mathrm{im}$ proving gut health under inflammatory conditions. ${ }^{83}$ The exoskeleton of the BSF prepupae contains the polysaccharide chitin $\left(\sim 87.0 \mathrm{~g} \mathrm{~kg}^{-1}, \mathrm{DM}\right)$, which might affect the digestibility and the utilization of other nutrients. ${ }^{84,85}$ In addition, high chitin inclusion in aquafeeds may induce intestinal inflammation and a reduction in fish welfare and growth. ${ }^{11,21}$

In this regard, in the present study, no drastic signs of intestinal inflammation were observed. A high supplementation of medium-chain fatty acids through the insect diet, possibly avoided the incidence of intestinal mucosa inflammation in fish larvae because of their important role in improving gut health. These data were also supported by molecular analysis on stress markers that did not show significant variations in their expression during the experiment.

Chitin has also been reported to decrease feed intake and growth in carp, tilapia (Oreochromis niloticus $\times O$. aureus), and Atlantic salmon at inclusion levels of $1 \%$ and higher, ${ }^{85-87}$ whereas no significant effects were observed in Oreochromis mossambicus fed a $60 \%$ insect meal or in eel (Anguilla japonica), red sea bream (Pagrus major), and yellowtail kingfish (Seriola quinqueradiata) fed a $10 \%$ chitin-supplemented diet. ${ }^{88,89}$ The differences observed in the abovementioned species may be explained by the chitinolytic activity of the single fish species. Some species, like turbot, do not have any chitinolytic activity during the early life stages, ${ }^{21}$ whereas others, like cobia, showed very high endochitinolytic activity. ${ }^{90}$ In cod (Gadus morhua) the enzyme chitinase was found in the stomach and intestinal tract, ${ }^{91-94}$ but no chitinolytic bacterial activity was reported. These differences are mainly related to the presence/absence of mechanical structures in fish larvae, able to break down the chitin exoskeleton of preys. Generally, the analysis of the six zebrafish chitinases in larvae fed different diets showed a time-dependent gene expression and pointed out that fish endogenous chitinase gene expression was not related to the amount of chitin provided through the diet. However, this result was not obvious, and opens new hypothesis about a possible involvement of intestinal bacteria in chitin digestion in fish.

\section{Conclusions}

The present study opens the possibility for the use of a more sustainable ingredient in the larval rearing of zebrafish. Knowledge obtained using experimental models such as zebrafish are becoming of primary importance for the development of the aquaculture industry. However, further studies are necessary to better understand fish responses and to evaluate possible effects on the different stages of the fish life cycle. In addition, lower insect meal inclusions should be considered for further studies because of their possible role as bioactive molecules and immunomodulators.

\section{Acknowledgment}

Funding was provided by Fondi di Ateneo 2017 to IO and $\mathrm{PhD}$ program 2016 to $\mathrm{A}$. $\mathrm{V}$.

\section{Disclosure Statement}

No competing financial interests exist.

\section{References}

1. Tacon AGJ, Metian M. Feed matters: satisfying the feed demand of aquaculture. Rev Fish Sci Aquac 2015;23:1-10.

2. FAO: The State of World Fisheries and Aquaculture 2016. Contributing to Food Security and Nutrition for All. FAO, Rome, 2016.

3. Natale F, Hofherr J, Fiore G, Virtanen J. Interactions between aquaculture and fisheries. Mar Policy 2013;38:205213.

4. Turchini GM, Ng W-K, Tocher DR: Fish Oil Replacement and Alternative Lipid Sources in Aquaculture Feeds. CRC Press, Taylor \& Francis group, USA, 2011.

5. Le François NR, Otton D, Werstink G: Finfish Aquaculture Diversification. CABI, United Kingdom, 2010.

6. Ayadi FY, Rosentrater K, Muthukumarappan K. Alternative protein sources for aquaculture feeds. J Aquacultre Feed Sci Nutr 2012;4:1-26.

7. Francis G, Makkar HPS, Becker K. Antinutritional factors present in plant-derived alternate fish feed ingredients and their effects in fish. Aquaculture 2001;199:197-227.

8. Tulli F, Chini Zittelli G, Giorgi G, Poli BM, Tibaldi E, Tredici MR. Effect of the Inclusion of Dried Tetraselmis suecica on growth, feed utilization, and fillet composition of european sea bass juveniles fed organic diets. J Aquat Food Prod Technol 2012;21:188-197.

9. Tibaldi E, Chini Zittelli G, Parisi G, Bruno M, Giorgi G, Tulli $\mathrm{F}$, et al. Growth performance and quality traits of European sea bass (D. labrax) fed diets including increasing levels of freeze-dried Isochrysis sp. (T-ISO) biomass as a source of protein and n-3 long chain PUFA in partial substitution of fish derivates. Aquaculture 2015;440:60-68.

10. Makkar HPS, Tran G, Heuzé V, Ankers P. State-of-the-art on use of insects as animal feed. Anim Feed Sci Technol 2014;197:1-33.

11. Henry M, Gasco L, Piccolo G, Fountoulaki E. Review on the use of insects in the diet of farmed fish: past and future. Anim Feed Sci Technol 2015;203:1-22.

12. Vogel H, Müller A, Heckel DG, Gutzeit H, Vilcinskas A. Nutritional immunology: diversification and diet-dependent expression of antimicrobial peptides in the black soldier fly Hermetia illucens. Dev Comp Immunol 2018;78:141-148. 
13. Cutrignelli MI, Messina M, Tulli F, Randazzo B, Olivotto I, Gasco L, et al. Evaluation of an insect meal of the Black Soldier Fly (Hermetia illucens) as soybean substitute: intestinal morphometry, enzymatic and microbial activity in laying hens. Res Vet Sci 2018;117:209-215.

14. Salomone R., Saija G, Mondello G, Giannetto A, Fasulo S, Davastano D. Environmental impact of food waste bioconversion by insects: application of life cycle assessment to process using Hermetia illucens. J Clean Prod 2017;140: 890-905.

15. Ramos-Elorduy J. Energy supplied by edible insects from Mexico and their nutritional and ecological importance. Ecol Food Nutr 2008;47:280-297.

16. Barroso FG, de Haro C, Sánchez-Muros MJ, Venegas E, Martínez-Sánchez A, Pérez-Bañón C. The potential of various insect species for use as food for fish. Aquaculture 2014;422-423:193-201.

17. Pimentel AC, Montali A, Bruno D, Tettamanti G. Metabolic adjustment of the larval fat body in Hermetia illucens to dietary conditions. J Asia Pac Entomol 2017;20: 1307-1313.

18. St-Hilaire S, Cranfill K, McGuire M. Fish offal recycling by the black soldier fly produces a foodstuff high in omega3 fatty acids. J World 2007;38:309-313.

19. Maciel-Vergara G, Ros VID. Viruses in insects for food and feed. J Invertebr Pathol 2017;147:60-75.

20. Poma G, Cuykx M, Amato E, Calaprice C, Focant JF, Covaci A. Evaluation of hazardous chemicals in edible insects and insect-based food intended for human consumption. Food Chem Toxicol 2017;100:70-79.

21. Kroeckel S, Harjes AGE, Roth I, Katz H, Wuertz S, Susenbeth A, et al. When a turbot catches a fly: evaluation of a pre-pupae meal of the black soldier fly (Hermetia illucens) as fish meal substitute - Growth performance and chitin degradation in juvenile turbot (Psetta maxima). Aquaculture 2012;364-365:345-352.

22. Feng W, Qian L, Wang W, Wang T, Deng Z, Yang F, et al. Exploring the potential of lipids from black soldier fly: new paradigm for biodiesel production (II) - Extraction kinetics and thermodynamic. Renew Energy 2018;119:12-18.

23. Diener S, Zurbrügg C, Gutiérrez FR, Nguyen DH, Morel A, Koottatep T, et al. Black soldier fly larvae for organic waste treatment-prospects and constraints. Proc WasteSafe 20112nd Int Conf Solid Waste Manag Dev Ctries 2011;52:1-8.

24. Chemello G, Piccinetti C, Randazzo B, Carnevali O, Maradonna F, Magro M, et al. Oxytetracycline delivery in adult female zebrafish by iron oxide nanoparticles. Zebrafish 2016;13:495-503.

25. Randazzo B, Chemello G, Tortarolo I, Chiarello GL, Zalas M, Santini A, et al. A novel photocatalytic purification system for fish culture. Zebrafish 2017;14:411-421.

26. Johnston IA, MacQueen DJ, Watabe S: Molecular biotechnology of development and growth in fish muscle. In: Fisheries for Global Welfare and Environment: Memorial Book of the 5th World Fisheries Congress 2008. Tsukamoto K, Kawamura T, Takeuchi T, Beard TD, \& Kaiser MJ (eds), pp. 241-262, Terrapub, Scotland, 2008.

27. De-Santis C, Jerry DR. Candidate growth genes in finfishWhere should we be looking? Aquaculture 2007;272:2238.

28. Dahm R, Geisler R. Learning from small fry: the zebrafish as a genetic model organism for aquaculture fish species. Mar Biotechnol 2006;8:329-345.
29. Aleström P, Holter JL, Nourizadeh-Lillabadi R. Zebrafish in functional genomics and aquatic biomedicine. Trends Biotechnol 2006;24:15-21.

30. Gobbi P, Martinez-Sanchez A, Rojo S. The effects of larval diet on adult life-history traits of the black soldier fly, Hermetia illucens (Diptera: Stratiomyidae). Eur J Entomol 2013;110:461-468.

31. Falcinelli S, Picchietti S, Rodiles A, Cossignani L, Merrifield DL, Taddei AR, et al. Lactobacillus rhamnosus lowers zebrafish lipid content by changing gut microbiota and host transcription of genes involved in lipid metabolism. Sci Rep 2015;5:8-10.

32. Olivotto I, Yasumasu S, Gioacchini G, Maradonna F, Cionna C, Carnevali O. Cloning and expression of high choriolytic enzyme, a component of the hatching enzyme system, during embryonic development of the marine ornamental fish Chrysiptera parasema. Mar Biol 2004;145: $1235-1241$.

33. Lawrence C, Adatto I, Best J, James A, Maloney K. Generation time of zebrafish (Danio rerio) and medakas (Oryzias latipes) housed in the same aquaculture facility. Lab Anim (NY) 2012;41:158-165.

34. Truzzi C, Illuminati S, Annibaldi A, Antonucci M, Scarponi G. Quantification of fatty acids in the muscle of antarctic fish Trematomus bernacchii by gas chromatographymass spectrometry: optimization of the analytical methodology. Chemosphere 2017;173:116-123.

35. Illuminati S, Truzzi C, Annibaldi A, Migliarini B, Carnevali $\mathrm{O}$, Scarponi $\mathrm{G}$. Cadmium bioaccumulation and metallothionein induction in the liver of the Antarctic teleost Trematomus bernacchii during an on-site short-term exposure to the metal via seawater. Toxicol Environ Chem 2010; 92:617-640.

36. Truzzi C, Annibaldi A, Illuminati S, Finale C, Scarponi G. Determination of proline in honey: comparison between official methods, optimization and validation of the analytical methodology. Food Chem 2014;150:477-481.

37. Truzzi C, Illuminati S, Finale C, Annibaldi A, Lestingi C, Scarponi G. Microwave-assisted solvent extraction of melamine from seafood and determination by Gas Chromatography-Mass Spectrometry: optimization by factorial design. Anal Lett 2014;47:1118-1133.

38. Carnevali O, Conti C, Ferraris P, Garavaglia MG, Gioacchini G, Giorgini E, et al. FT-IR Microspectroscopy on molecular building of zebrafish oocytes. J Mol Struct 2009; 938:207-213.

39. Gioacchini G, Giorgini E, Olivotto I, Maradonna F, Merrifield DL, Carnevali O. The influence of probiotics on zebrafish Danio Rerio innate immunity and hepatic stress. Zebrafish 2014;11:98-106.

40. Giorgini E, Gioacchini G, Conti C, Ferraris P, Sabbatini S, Tosi G, et al. The role of melatonin on zebrafish follicle development: an FT-IR imaging approach. Vib Spectrosc 2012;62:279-285.

41. Zuur A, Leno EN, Smith GN: Analyzing Ecological Data. Springer-Verlag, New York, 2007.

42. Giorgini E, Sabbatini S, Conti C, Rubini C, Rocchetti R, Re $\mathrm{M}$, et al. Vibrational mapping of sinonasal lesions by Fourier transform infrared imaging spectroscopy. J Biomed Opt 2015;20:125003.

43. Sato K, Seimiya M, Kodera Y, Kitamura A, Nomura F. Application of Fourier-transform infrared (FT-IR) spectroscopy for simple and easy determination of chylomicron- 
triglyceride and very low density lipoprotein-triglyceride. Clin Chim Acta 2010;411:285-290.

44. Stuart BH: Infrared Spectroscopy: Fundamentals and Applications. J. Wiley \& Sons, United Kingdom, 2004.

45. Movasaghi Z, Rehman S, ur Rehman DI. Fourier transform infrared (FTIR) spectroscopy of biological tissues. Appl Spectrosc Rev 2008;43:134-179.

46. Rudbeck ME, Kumar S, Mroginski M-A, Lill SON, Blomberg MRA, Barth A. Infrared spectrum of phosphoenol pyruvate: computational and experimental studies. J Phys Chem A 2009;113:2935-2942.

47. Belbachir K, Noreen R, Gouspillou G, Petibois C. Collagen types analysis and differentiation by FTIR spectroscopy. Anal Bioanal Chem 2009;395:829-837.

48. Khajehpour M, Dashnau JL, Vanderkooi JM. Infrared spectroscopy used to evaluate glycosylation of proteins. Anal Biochem 2006;348:40-48.

49. Caligiani A, Marseglia A, Leni G, Baldassarre S, Maistrello L, Dossena A, et al. Composition of black soldier fly prepupae and systematic approaches for extraction and fractionation of proteins, lipids and chitin. Food Res Int 2018; 105:812-820.

50. Bondari K, Sheppard DC. Soldier fly larvae as feed in commercial fish production. Aquaculture 1981;24:103-109.

51. Elwert C, Knips I, Katz P: A novel protein source: maggot meal of the black soldier fly (Hermetia illucens) in broiler feed. In: 11. Tagung Schweine- und Geflügelernährung, Germany. Gierus M, Kluth H, Bulang M \& Kluge H (eds), Lutherstadt, Wittenberg, 2010.

52. Newton GL, Booram CV, Barker RV, Hale OM. Dried Hermetia Illucens larvae meal as a supplement for swine. J Anim Sci 1997;40:385-400.

53. Stamer A, Katz P, Wessel S, Hörstgen-Schwark G: Protein Concentrates Derived from Fly Mass-Production: HermetiaMeal as an Alternative to Fishmeal. 16th IFOAM Org World Congr 2008; pp. 16-20, Modena, Italy.

54. Bruni L, Pastorelli R, Viti C, Gasco L, Parisi G. Characterisation of the intestinal microbial communities of rainbow trout (Oncorhynchus mykiss) fed with Hermetia illucens (black soldier fly) partially defatted larva meal as partial dietary protein source. Aquaculture 2018;487:56-63.

55. Lawrence $\mathrm{C}$. The husbandry of zebrafish (Danio rerio): a review. Aquaculture 2007;269:1-20.

56. Dutta S. Food and feeding habits of Danio rerio (Ham. Buch) inhabiting Gadigarh Stream, Jammu. J Freshw Biol 1993;52:165-168.

57. McClure MM, McIntyre PB, McCune AR. Notes on the natural diet and habitat of eight danionin fishes, including the zebrafish Danio rerio. J Fish Biol 2006;69: 553-570.

58. Spence R, Fatema MK, Ellis S, Ahmed ZF, Smith C. Diet, growth and recruitment of wild zebrafish in Bangladesh. J Fish Biol 2007;71:304-309.

59. Gasco L, Henry M, Piccolo G, Marono S, Gai F, Renna M, et al. Tenebrio molitor meal in diets for European sea bass (Dicentrarchus labrax L.) juveniles: growth performance, whole body composition and in vivo apparent digestibility. Anim Feed Sci Technol 2016;220:34-45.

60. Piccolo G, Iaconisi V, Marono S, Gasco L, Loponte R, Nizza S, et al. Effect of Tenebrio molitor larvae meal on growth performance, in vivo nutrients digestibility, somatic and marketable indexes of gilthead sea bream (Sparus aurata). Anim Feed Sci Technol 2017;226:12-20.
61. Lock ER, Arsiwalla T, Waagbo R. Insect larvae meal as an alternative source of nutrients in the diet of Atlantic salmon (Salmo salar) postsmolt. Aquac Nutr 2016;22:1202-1213.

62. Halver JE, Hardy RW: Fish Nutrition. Academic Press, San Diego: CA, 2002.

63. Sargent JR, McEvoy LA, Bell JG. Requirements, presentation and sources of polyunsaturated fatty acids in marine fish larval feeds. Aquaculture 1997;155:117-127.

64. Paul A, Frederich M, Megido RC, Alabi T, Malik P, Uyttenbroeck R, et al. Insect fatty acids: a comparison of lipids from three Orthopterans and Tenebrio molitor L. larvae. J Asia Pac Entomol 2017;20:337-340.

65. Liland NS, Biancarosa I, Araujo P, Biemans D, Bruckner $\mathrm{CG}$, Waagb $\varnothing \mathrm{R}$, et al. Modulation of nutrient composition of black soldier fly (Hermetia illucens) larvae by feeding seaweed-enriched media. PLoS One 2017;12:e0183188.

66. Seiliez I, Panserat S, Corraze G, Kaushik S, Bergot P. Cloning and nutritional regulation of a $\Delta 6$-desaturase-like enzyme in the marine teleost gilthead seabream (Sparus aurata). Comp Biochem Physiol Part B Biochem Mol Biol 2003;135:449-460.

67. Robin J H, Skalli A. Incorporation of dietary fatty acid in European sea bass (Dicentrarchus labrax) - A methodological approach evidencing losses of highly unsaturated fatty acids. Aquaculture 2007;263:227-237.

68. Sealey WM, Gaylord TG, Barrows FT, Tomberlin JK, McGuire MA, Ross C, et al. Sensory analysis of rainbow trout, Oncorhynchus mykiss, fed enriched black soldier fly prepupae, Hermetia illucens. J World Aquac Soc 2011; 42:34-45.

69. Oonincx DGAB, Van Broekhoven S, Van Huis A, Van Loon JJA. Feed conversion, survival and development, and composition of four insect species on diets composed of food by-products. PLoS One 2015;10:1-20.

70. Dayrit FM. The Properties of lauric acid and their significance in coconut oil. J Am Oil Chem Soc 2015;92:1-15.

71. Hashim SA, Tantibhedyangkul P. Medium chain triglyceride in early life: effects on growth of adipose tissue. Lipids 1987;22:429-434.

72. Lavau MM, Hashim SA. Effect of medium chain triglyceride on lipogenesis and body fat in the rat. J Nutr 1978; 108:613-620.

73. Li S, Ji H, Zhang B, Tian J, Zhou J, Yu H. Influence of black soldier fly (Hermetia illucens) larvae oil on growth performance, body composition, tissue fatty acid composition and lipid deposition in juvenile Jian carp (Cyprinus carpio var. Jian). Aquaculture 2016;465:43-52.

74. Davis DA, Lazo JP, Arnold CR. Response of juvenile red drum (Sciaenops ocellatus) to practical diets supplemented with medium chain triglycerides. Fish Physiol Biochem 1999; 21:235-248.

75. Turchini GM, Torstensen BE, Ng W-K. Fish oil replacement in finfish nutrition. Rev Aquac 2009;1:10-57.

76. Landgraf K, Schuster S, Meusel A, Garten A, Riemer T, Schleinitz D, et al. Short-term overfeeding of zebrafish with normal or high-fat diet as a model for the development of metabolically healthy versus unhealthy obesity. BMC Physiol 2017; 17:4.

77. Lecomte M, Couëdelo L, Meugnier E, Plaisancié P, Létisse $\mathrm{M}$, Benoit B, et al. Dietary emulsifiers from milk and soybean differently impact adiposity and inflammation in association with modulation of colonic goblet cells in highfat fed mice. Mol Nutr Food Res 2016;60:609-620. 
78. Benoit B, Plaisancié P, Géloën A, Estienne M, Debard C, Meugnier E, et al. Pasture v. standard dairy cream in highfat diet-fed mice: improved metabolic outcomes and stronger intestinal barrier. Br J Nutr 2014;112:1-16.

79. Gulhane M, Murray L, Lourie R, Tong H, Sheng YH, Wang R, et al. High fat diets induce colonic epithelial cell stress and inflammation that is reversed by IL-22. Sci Rep 2016;6:1-17.

80. Urán PA, Schrama JW, Rombout JHWM, Obach A, Jensen L, Koppe W, et al. Soybean meal-induced enteritis in Atlantic salmon (Salmo salar L.) at different temperatures. Aquac Nutr 2008;14:324-330.

81. Harkins RW, Sarett HP. Medium-chain triglycerides. JAMA 1968;203:272-274.

82. Liu Y. Fatty acids, inflammation and intestinal health in pigs. J Anim Sci Biotechnol 2015;6:41.

83. Bertevello PL, De Nardi L, Torrinhas RS, Logullo AF, Waitzberg DL. Partial replacement of $\omega-6$ fatty Acids with medium-chain triglycerides, but not olive Oil, improves colon cytokine response and damage in experimental colitis. J Parenter Enter Nutr 2012;36:442-448.

84. Diener S, Zurbrügg C, Tockner K. Conversion of organic material by black soldier fly larvae: establishing optimal feeding rates. Waste Manag Res 2009;27:603-610.

85. Shiau S-Y, Yu Y-P. Dietary supplementation of chitin and chitosan depresses growth in tilapia, Oreochromis niloticus $\times$ O. aureus. Aquaculture 1999;179:439-446.

86. Gopalakannan A, Arul V. Immunomodulatory effects of dietary intake of chitin, chitosan and levamisole on the immune system of Cyprinus carpio and control of Aeromonas hydrophila infection in ponds. Aquaculture 2006;255: 179-187.

87. Hansen A-C, Rosenlund G, Karlsen $\varnothing$, Koppe W, Hemre G-I. Total replacement of fish meal with plant proteins in diets for Atlantic cod (Gadus morhua L.) I-Effects on growth and protein retention. Aquaculture 2007;272: 599-611.

88. Rapatsa MM, Moyo NAG. Evaluation of Imbrasia belina meal as a fishmeal substitute in Oreochromis mossambicus diets: growth performance, histological analysis and enzyme activity. Aquac Reports 2017;5:18-26.

89. Kono M, Matsui T, Shimizu C. Effect of chitin, chitosan, and cellulose as diet supplements on the growth of cultured fish. Nippon Suisan Gakkaish 1987;53:125-129.

90. Fines BC, Holt GJ. Chitinase and apparent digestibility of chitin in the digestive tract of juvenile cobia, Rachycentron canadum. Aquaculture 2010;303:34-39.

91. Danulat E. The effects of various diets on chitinase and $\beta$ glucosidase activities and the condition of cod, Gadus morhua (L.). J Fish Biol 1986;28:191-197.

92. Danulat E. Role of bacteria with regard to chitin degradation in the digestive tract of the cod (Gadus morhua). Mar Biol 1986;90:335-343.

93. Danulat E, Kausch H. Chitinase activity in the digestive tract of the cod Gadus morhua (L.). J Fish Biol 1984;24.

94. Lindsay GJH, Walton MJ, Adron JW, Fletcher TC, Cho $\mathrm{CY}$, Cowey CB. The growth of rainbow trout (Salmo gairdneri) given diets containing chitin and its relationship to chitinolytic enzymes and chitin digestibility. Aquaculture 1984;37:315-334.

Address correspondence to:

Ike Olivotto, PhD

Dipartimento di Scienze della Vita e dell'Ambiente Università Politecnica delle Marche via Brecce Bianche Ancona 60131 Italy

E-mail: i.olivotto@univpm.it 